\title{
Spatial Direct Numerical Simulation of Boundary-Layer \\ Transition Mechanisms: Validation of PSE Theory
}

\author{
R.D. Joslin and C.L. Streett \\ NASA Langley Research Center, Hampton, VA 23681 \\ and \\ C.-L. Chang \\ High Technology Corporation, Hampton, VA 23666
}

\begin{abstract}
A study of instabilities in incompressible boundary-layer flow on a flat plate is conducted by spatial direct numerical simulation (DNS) of the Navier-Stokes equations. Here, the DNS results are used to critically evaluate the results obtained using parabolized stability equations (PSE) theory and to study mechanisms associated with breakdown from laminar to turbulent flow. Three test cases are considered: two-dimensional TollmienSchlichting wave propagation, subharmonic instability breakdown, and oblique-wave breakdown. The instability modes predicted by PSE theory are in good quantitative agreement with the DNS results, except a small discrepancy is evident in the mean-flow distortion component of the 2-D test problem. This discrepancy is attributed to far-field boundarycondition differences. Both DNS and PSE theory results show several modal discrepancies when compared with the experiments of subharmonic breakdown. Computations that allow for a small adverse pressure gradient in the basic flow and a variation of the disturbance frequency result in better agreement with the experiments.
\end{abstract}




\section{Introduction}

In the past century, numerous attempts have been made to understand and predict the transition from laminar to turbulent flow in boundary layers. Most of this effort stems from the independent theoretical accomplishments of Orr (1907) and Sommerfeld (1908) at the turn of the 20th century. The Orr-Sommerfeld equation is based on linearized disturbance equations and is a successful example of classical hydrodynamic stability theory. Nearly 20 years later Tollmien (1929) solved the Orr-Sommerfeld equation, which led to the calculation of a critical Reynolds number for the onset of instability. On the same road, Schlichting (1933) computed amplification rates of disturbances in the boundary layer. The first experimental confirmation of the theory was accomplished by Schubauer and Skramstad (1943, 1947); a vibrating ribbon was used to impress a disturbance into the boundary layer, and hot wires were used for measurements. These contributions (and others) span over some 40 years of research, after which theory and experiments finally agree on the initial growth of disturbances in boundary layers.

Stability theory has gained wide acceptance and is now a well-established tool in the research and engineering community. The first reasonably comprehensive method for predicting transition was derived from stability theory: the $e^{N}$ method by Smith and Gamberoni (1956) and van Ingen (1956). Although the $e^{N}$ method is widely used to predict transition in a broad class of flows, this method has considerable shortcomings. Because the approach uses linear stability theory, the limitations of the theory are imbedded in the methodology. Specifically, a quasi-parallel boundary layer is assumed, and because the equations are linear, no amplitude information about the ingested disturbance can be taken into account. Finally, the method is semi-empirical; therefore, some foreknowledge is required of the flow in transition. The true physical problem involves disturbances that interact in a nonlinear manner in later stages of transition and are imbedded in a growing boundary layer. Consequently, a method that accounts for nonparallel flow and nonlinear interactions is necessary to predict 
transition. At present, such an all-encompassing method of transition prediction is beyond our grasp; however, progress has been made in recent years.

In the last decade, significant accomplishments have been made in the theoretical prediction of transition beyond the linear growth stage. Based, in part, on pioneering attempts at nonlinear theories (e.g., Benney and Lin, 1960; Craik, 1971), Orszag and Patera (1980, 1983 ) by numerical experiments and Herbert (1984) by theory discovered a secondary instability mechanism. Herbert's (1984) theory is based on Floquet theory and accounts for an experimentally observed three-dimensional (3-D) instability. Although the governing equations are linearized and a local parallel-flow assumption is made, remarkable agreement is obtained between predictions from Herbert's theory and experimental results, particularly for the peak-valley splitting mode identified by Klebanoff et al. (1959, 1962) and for the peak-valley alignment mode observed by Kachanov et al. (1977, 1984). These modes are two distinct and different routes to transition that are discriminated based on the initial disturbance levels. After nearly a decade of verification, Herbert's theory for secondary instabilities is generally accepted and widely used by the research community to understand certain aspects of transition in boundary layers.

More recently, Herbert and Bertolotti (1987) have devised a nonlinear, nonparallel computational method based on so-called parabolized stability equations (PSE). The benefits and limitations of this new theory have yet to be determined and will be explored somewhat in the present paper. Before the development of this theory, the only approach to solve the nonparallel, nonlinear boundary-layer transition problem was by direct numerical simulation (DNS), except in the large Reynolds number limit where asymptotic methods have had some success (e.g., Smith, 1979; Hall and Smith, 1991). To date, most studies using DNS have been limited to the temporal formulation-where a spatially-periodic computational domain travels with the disturbance and the temporal evolution of the disturbance is computed. This enabled simulations into the later stages of transition (Zang and Hussaini, 
1987,1990; and Laurien and Kleiser, 1989), thus providing a database of qualitative information, which, however, lacks the physically-realistic spatial representation. Spatial DNS computes spatially-evolving disturbances and would provide needed quantitative information about transition. Progress in spatial DNS has been made by, among others, Danabasoglu et al. (1990, 1991) for channel flow and Fasel (1976), Spalart (1989), Fasel et al. (1990), Rai and Moin (1991a, 1991b), and Joslin et al. (1992a) for boundary-layer flow. Results obtained from spatial DNS have been compared qualitatively and, with some success, quantitatively to linear stability theory, secondary instability theory, and available experimental results. For a more complete list of accomplishments in transition prediction with DNS, refer to the review by Kleiser and Zang (1991).

The goal of the present research is to quantify various transitional-flow mechanisms of interest from the linear region to the nonlinear breakdown stage and to provide a critical comparison of spatial DNS and PSE theory results. To date, this comparison offers the most rigorous test of the PSE approach for accuracy; the main focus will be to point out strengths and potential weaknesses of PSE theory and the impact of these weaknesses on the overall flow-field prediction. To accomplish this goal, three test cases are computed by spatial DNS and then compared to PSE theory: two-dimensional (2-D) Tollmien-Schlichting (TS) wave propagation, subharmonic breakdown, and oblique-wave breakdown.

\section{Governing Equations}

To compute the disturbance development, the incompressible Navier-Stokes equations are solved. The streamwise direction is $x$, the wall-normal direction is $y$, and the spanwise

direction is $z$. Instantaneous velocities $\underline{\tilde{u}}=(\tilde{u}, \tilde{v}, \tilde{w})$ and pressure $\tilde{p}$ are decomposed into the basic components $\underline{U}=(U, V, W)$ and $P$ and the disturbance components $\underline{u}=(u, v, w)$ and $p$ so that

$$
\underline{\tilde{u}}(\underline{x}, t)=\underline{U}(\underline{x})+\underline{u}(\underline{x}, t) \quad \text { and } \quad \tilde{p}(\underline{x}, t)=P(\underline{x})+p(\underline{x}, t)
$$

where $\underline{x}=(x, y, z)$ and $t$ is time. 
The basic flow on a flat plate is generally governed by the full Navier-Stokes equations. However, according to order-of-magnitude analysis, the streamwise parabolized form of the equations (boundary-layer equations) is adequate. A marching algorithm may be used to solve the equations for the basic flow $(U, V)$, or the widely used Blasius similarity profile can be employed. These approaches have been verified by comparisons with experiments (see Schlichting, 1955). For the present study, the Blasius flow is used for the basic state.

To determine the disturbance component of equation (1), the equations that are solved result from substituting equation (1) into the Navier-Stokes equations and subtracting the basic flow equations. These unsteady, nonlinear disturbance equations are

$$
\frac{\partial \underline{u}}{\partial t}+(\underline{u} \cdot \nabla) \underline{u}+(\underline{U} \cdot \nabla) \underline{u}+(\underline{u} \cdot \nabla) \underline{U}=-\nabla p+\frac{1}{R_{\delta_{o}^{*}}} \nabla^{2} \underline{u}
$$

and the continuity equation

$$
\nabla \cdot \underline{u}=0
$$

The equations are nondimensionalized with respect to the freestream velocity $U_{\infty}$, the kinematic viscosity $\nu$, and some length scale at the inflow (e.g., displacement thickness $\delta_{0}^{*}$ ). A Reynolds number can then be defined $R_{\delta_{o}^{*}}=U_{\infty} \delta_{o}^{*} / \nu$.

At the wall and in the far field, boundary conditions for equations (2) and (3) are

$$
\underline{u}=0 \quad \text { at } \quad y=0 \quad \text { and } \quad \underline{u} \rightarrow 0 \quad \text { as } \quad y \rightarrow \infty
$$

To prevent spurious reflections, outflow conditions are provided by the buffer-domain technique (Streett and Macaraeg, 1989; Joslin et al. 1992a). For the present study, the disturbance forcing takes the form of eigenfunctions that are imposed at the inflow boundary. Controlled disturbances at the inflow are required to facilitate the comparison. To this end, the inflow condition for both DNS and PSE is given by the basic flow plus the disturbance forcing functions

$$
\underline{u}_{o}=\underline{U}_{o}+\sum_{n=-N_{z}}^{N_{z}} \sum_{m=-N_{t}}^{N_{t}} A_{m, n}^{o} \cdot\left[\underline{\hat{u}}_{m, n}^{o}(y) \exp [i(n \beta z-m \omega t)]\right]
$$


where $\underline{U}_{o}$ is the inflow basic component; $A_{m, n}^{o}$ are prescribed $2-\mathrm{D}$ and $3-\mathrm{D}$ disturbance am-

plitudes; $\beta$ is an imposed spanwise wave number; and $\omega$ is an imposed disturbance frequency. The terms $\underline{\hat{u}}_{m, n}^{o}(y)$ are complex eigenfunctions found either by solving the Orr-Sommerfeld and Squire equations or obtained from a secondary instability theory (see Herbert, 1984). The eigenfunctions $\underline{\hat{u}}_{m, n}^{o}(y)$ are normalized with respect to the maximum streamwise-velocity component such that the initial amplitudes of the induced disturbances are prescribed by $A_{m, n}^{o}$

\section{Computational Methods of Solution}

In this paper, spatial DNS is used to study breakdown mechanisms occurring in transitional flows, and to evaluate the accuracy of PSE theory in predicting the evolution of convective disturbances. A description of both the DNS and PSE theory approaches follows.

\section{Spatial DNS.}

In a previous study by the authors described in Joslin et al. (1992a), the spatial DNS approach involved fourth-order compact differences used on a nonstaggered grid. For this study, sixth-order compact-difference schemes have replaced the fourth-order schemes, and a staggered grid has replaced the nonstaggered-grid formulation.

In the streamwise direction ( $x$-direction), fourth-order central finite differences are used for the pressure equation. At boundary and near-boundary nodes, fourth-order differences are used. For first and second derivatives in the momentum equations, sixth-order compact differences are used. As described by Lele (1992), the difference equations are

$$
\frac{1}{3} f_{i-1}^{\prime}+f_{i}^{\prime}+\frac{1}{3} f_{i+1}^{\prime}=\frac{7}{9 h_{x}}\left(f_{i+1}-f_{i-1}\right)+\frac{1}{36 h_{x}}\left(f_{i+2}-f_{i-2}\right)
$$

and

$$
\frac{2}{11} f_{i-1}^{\prime \prime}+f_{i}^{\prime \prime}+\frac{2}{11} f_{i+1}^{\prime \prime}=\frac{12}{11 h_{x}^{2}}\left(f_{i+1}-2 f_{i}+f_{i-1}\right)+\frac{3}{44 h_{x}^{2}}\left(f_{i+2}-2 f_{i}+f_{i-2}\right)
$$

where $h_{x}$ is the uniform streamwise step size, and $f$ is an arbitrary function whose derivatives are sought. At boundary and near-boundary nodes, explicit fifth-order finite differences are 
used. Equations (6) and (7) lead to tridiagonal systems which can be solved efficiently by LU-decomposition with appropriate backward and forward substitutions.

In the wall-normal direction ( $y$-direction), Chebyshev series are used to approximate the disturbance at Gauss-Lobatto collocation points. Since this series and its associated spectral operators are defined on $[-1,1]$ and the physical problem of interest has a semi-infinite domain $[0, \infty)$ or a truncated domain $\left[0, y_{\max }\right]$, a transformation is employed. Studies of spectral methods and mapping tranformations in unbounded regions have been conducted by Grosch and Orszag (1977) and Boyd(1989). Here an algebraic mapping is used:

$$
y=\frac{y_{\max } s_{p}(1+\bar{y})}{2 s_{p}+y_{\max }(1-\bar{y})} \quad \text { or } \quad \bar{y}=\frac{\left(2 s_{p}+y_{\max }\right) y-y_{\max } s_{p}}{y_{\max }\left(s_{p}+y\right)}
$$

where $y \in\left[0, y_{\max }\right)$ and $\bar{y} \in[-1,1] ; y_{\max }$ is the wall-normal distance from the wall to the farfield boundary in the truncated domain, and $s_{p}$ controls the grid stretching in the wall-normal direction. In the spanwise direction ( $z$-direction), periodicity is assumed, allowing for Fourier series representations. Using Fourier series, spectral accuracy is obtained in the spanwise direction and Fast Fourier Transforms (FFT) or sine/cosine transforms may be used, allowing for the fast computing of derivatives. For more details on the spectral methods used here refer to Canuto et al. (1988) and Joslin et al. (1992a). Although spectral accuracy is obtained in the wall-normal and spanwise directions, the overall spatial-discretization scheme is formally fourth-order accurate as a result of the streamwise discretization.

For time marching, a time-splitting procedure was used with implicit second-order Crank-Nicolson differencing for normal diffusion terms; an explicit third-order three-stage Runge-Kutta (RK) method was used for the remaining terms. This time-stepping procedure was used successfully by Streett and Hussaini (1991) for Taylor-Couette flow simulations. The pressure is omitted from the momentum equations (2) for the fractional RK stage, leading to

$$
\frac{\underline{u}^{*}-\underline{u}^{m}}{h_{t}^{m}}=C_{1}^{m} H^{m}(\underline{u})+\frac{C_{2}^{m}}{R_{\delta_{o}^{*}}} D^{2}\left(\underline{u}^{*}+\underline{u}^{m}\right),
$$


where

$$
H^{m}(\underline{u})=L(\underline{u})^{m}+C_{3}^{m} H^{m-1}(\underline{u})
$$

and operator

$$
L(\underline{u})=(\underline{U} \cdot \nabla) \underline{u}+(\underline{u} \cdot \nabla) \underline{U}+(\underline{u} \cdot \nabla) \underline{u}-\frac{1}{R_{\delta_{o}^{*}}} \nabla_{x z}^{2} \underline{u},
$$

Here $\underline{u}^{*}$ are disturbance velocities at the intermediate RK stages, $\underline{u}^{m}$ are velocities at previous RK stages $(m=1,2$ or 3$), \underline{u}^{o}$ are velocities at the previous time-step, $\nabla_{x z}^{2}=\partial^{2} / \partial x^{2}+\partial^{2} / \partial z^{2}$, $h_{t}$ is the time-step size, and $D$ is the wall-normal spectral derivative operator. For a full RK stage, the momentum equations with the pressure are

$$
\frac{\underline{u}^{m+1}-\underline{u}^{m}}{h_{t}^{m}}=C_{1}^{m} H^{m}(\underline{u})+\frac{C_{2}^{m}}{R_{\delta_{o}^{*}}} D^{2}\left(\underline{u}^{m+1}+\underline{u}^{m}\right)-\nabla p^{m+1}
$$

Subtracting (9) from (10) leaves

$$
\frac{\underline{u}^{m+1}-\underline{u}^{*}}{h_{t}^{m}}=-\nabla p^{m+1}
$$

By taking the divergence of (11) and imposing zero-divergence of the flow field at each RK stage, a pressure equation is obtained

$$
\nabla^{2} p^{m+1}=\frac{1}{h_{t}^{m}}\left(\nabla \cdot \underline{u}^{*}\right)
$$

which is subject to homogeneous Neumann boundary conditions. This boundary condition is justified in the context of a time-splitting scheme as discussed by Streett and Hussaini (1991). The solution procedure is as follows: The intermediate RK velocities $\left(\underline{u}^{*}\right)$ are determined by solving equation (9). The pressure correction $\left(p^{m+1}\right)$ is found by solving (12). Then, the full RK stage velocities $\left(\underline{u}^{m+1}\right)$ are obtained from (11). Upon solving the above system three consecutive times, full time-step $(n+1)$ velocities are determined, where $\underline{u}^{n+1}=\underline{u}^{m=3}$. The RK coefficients and time-steps given by Williamson (1980) are

$$
\left(\begin{array}{lll}
C_{1}^{1} & C_{2}^{1} & C_{3}^{1} \\
C_{1}^{2} & C_{2}^{2} & C_{3}^{2} \\
C_{1}^{3} & C_{2}^{3} & C_{3}^{3}
\end{array}\right)=\left(\begin{array}{ccc}
1 & 1 / 2 & 0 \\
9 / 4 & 1 / 2 & -4 \\
32 / 15 & 1 / 2 & -153 / 32
\end{array}\right) \quad \text { and } \quad\left\{\begin{array}{c}
h_{t}^{1} \\
h_{t}^{2} \\
h_{t}^{3}
\end{array}\right\}=\left\{\begin{array}{c}
1 / 3 h_{t} \\
5 / 12 h_{t} \\
1 / 4 h_{t}
\end{array}\right\}
$$


where the sum of the three RK time-steps equals the full time-step $\left(h_{t}\right)$.

A significant improvement in the spatial DNS approach over that used in Joslin et al. (1992) involves the use of staggered grids in the wall-normal direction. The velocities and the 2-D pressure component are computed at Gauss-Lobatto points as before, and the 3D pressure components are computed at Gauss points. With this staggered-grid pressure solver, reductions of 10-50 percent in CPU time, 30-50 percent in virtual memory, and 50-70 percent in run-time disk requirements were obtained, compared with the nonstaggered-grid solver. This staggered grid approach affects only the pressure equation (12) and is described in the remaining portion of this subsection.

To obtain the pressure correction $(p)$ for the 2-D and 3-D boundary-layer problems, solutions of Poisson equations for each RK stage are required. For 3-D simulations with spanwise periodicity assumed, the pressure correction is determined in transform space where the Fourier coefficients are evaluated. In transform space, the Poisson equations become Helmholtz equations. In order to solve the equations efficiently, a fast elliptic solver is required. For this purpose, the tensor-product method described by Lynch et al. (1964) is used. On a nonstaggered grid, this approach was employed by Danabasoglu et al. (1990, 1991) for the channel problem and by Joslin et al. (1992a) for the boundary-layer problem.

For the present staggered-grid formulation, the discretized equations for the 3-D components are

$$
\left(H-\beta_{n}^{2} I\right) p_{n}+p_{n} X^{T}=R_{n}
$$

where matrix operations are ordered with respect to $(y, x) \cdot p_{n}$ are Fourier coefficients of the desired pressure solution $p, \beta_{n}=n \beta$ are spanwise wave-number coefficients of the Fourier series, $I$ is the identity matrix, $X^{T}$ is the transpose of the streamwise central finite-difference operator, and $R_{n}$ are known Fourier coefficients of the right side of the pressure equation (12). The following matrix operations determine the wall-normal operator $H$ :

$$
H=I_{G L}^{G} D \tilde{D} I_{G}^{G L}
$$


where $D$ is a spectral wall-normal derivative operator for the stretched grid, and $\tilde{D}$ is the derivative matrix with the first and last rows set to zero. This modification enforces the homogeneous Neumann boundary conditions required for $p$ on the Gauss-Lobatto grid. The interpolation matrix $I_{G L}^{G}$ operates on variables at Gauss-Lobatto points and transforms them to Gauss points; the interpolation matrix $I_{G}^{G L}$ performs the inverse operation. These operators are defined as

$$
I_{G L}^{G}=\left(C_{G}^{-1}\right)_{j, k}\left(C_{G L}\right)_{k, j} \quad \text { and } \quad I_{G}^{G L}=\left(C_{G L}^{-1}\right)_{j, k}\left(C_{G}\right)_{k, j}
$$

where

$$
\left(C_{G}\right)_{k, j}=\frac{2}{\bar{N}_{y} c_{k}} \cos \left[\frac{\left(j+\frac{1}{2}\right) \pi k}{\bar{N}_{y}}\right], \quad\left(C_{G}^{-1}\right)_{j, k}=\cos \left[\frac{\left(j+\frac{1}{2}\right) \pi k}{\bar{N}_{y}}\right]
$$

with $j=0,1, \ldots, \bar{N}_{y}-1 ; k=0,1, \ldots, \bar{N}_{y} ;$ and

$$
\left(C_{G L}\right)_{k, j}=\frac{2}{\bar{N}_{y} c_{k} c_{j}} \cos \left[\frac{k j \pi}{\bar{N}_{y}}\right], \quad\left(C_{G L}^{-1}\right)_{j, k}=\cos \left[\frac{k j \pi}{\bar{N}_{y}}\right]
$$

with $j=0,1, \ldots, \bar{N}_{y}$; and $k=0,1, \ldots, \bar{N}_{y}$. The constants $c_{i}$ are defined as $c_{i}=1 ; i=$ $1,2, \ldots, \bar{N}_{y}-1 ;$ and $c_{0}=c_{\bar{N}_{y}}=2$.

Using the tensor-product method described by Lynch et al. (1964), the matrix $H$ may be decomposed into

$$
H=Q \Lambda Q^{-1}
$$

where $\Lambda$ is a diagonal matrix of eigenvalues and $Q$ is the corresponding matrix of eigenvectors. Temporary matrices are introduced and defined:

$$
\hat{p}_{n}=Q^{-1} p_{n} \quad \text { and } \quad G_{n}=Q^{-1} R_{n}
$$

Substituting (17) and (18) into (14), one obtains

$$
\left(\Lambda-\beta_{n}^{2}\right) \hat{p}_{n}+\hat{p}_{n} X^{T}=G_{n}
$$

Equation (19) is used to solve for $\hat{p}_{n}$, which is then used in (18) to solve for $p_{n}$. The solution is then inverse tranformed to physical space. The operators: $H, Q, Q^{-1}, \Lambda$ and 
$X^{T}$ are all mesh dependent matrices and need be calculated but once, as is the influence matrix. Since $X^{T}$ is a fourth-order accurate pentadiagonal matrix, the LU-decomposition method is used and provides and efficient method to solve for the pressure. The influencematrix technique is also used for the pressure solver to ensure that the continuity equation is discretely satisfied. Details of the influence-matrix technique are given by Streett and Hussaini (1991), Danabasoglu et al. (1991), and Joslin et al. (1992a). Since the pressure equation (12) is an inviscid calculation, it is well posed provided that boundary conditions on the normal component of velocity only are enforced. At the end of each full RK time-step, a non-zero tangential velocity component may therefore arise at the computational boundary. This is referred to as a "slip-velocity." To alleviate this problem, intermediate boundary conditions as described by Streett and Hussaini (1991) and Joslin et al. (1992a) are used and given by

$$
\underline{u}_{\tau}^{*}=\underline{u}_{B C}+h_{t}^{m}\left[\left(1+\frac{h_{t}^{m}}{h_{t}^{m-1}}\right) \nabla \wp_{\tau}^{m}-\frac{h_{t}^{m}}{h_{t}^{m-1}} \nabla \wp_{\tau}^{m-1}\right]
$$

where $\underline{u}_{B C}=0$ for a rigid wall and $\underline{u}_{B C}=\underline{u}_{0}$ for an inflow condition or a wall slot condition evaluated at the appropriate time in the RK stage.

Disturbances are introduced into the boundary layer by forcing at the inflow boundary. At the outflow, the buffer-domain technique of Streett and Macaraeg (1989) is used. Briefly, the streamwise convective terms are attenuated to zero in a small region that is appended to the end of the physical domain. As shown by Joslin et al. (1992a) for flat-plate boundarylayer flow, a buffer-domain length of approximately three streamwise wavelengths is adequate to provide an attenuation function that is smooth enough to avoid the reflection of waves. 


\section{PSE Theory.}

In contrast to the spatial DNS approach described above in which the complete NavierStokes equations are solved, the PSE approach seeks approximate solutions to the unsteady Navier-Stokes equations, which can be obtained by an efficient marching procedure. Generally, there are a number of ways to parabolize the Navier-Stokes equations; however, any acceptable approximation must be able to capture the physics of instability waves. One such method was first suggested by Herbert and Bertolotti (1987); more details of this method and a comprehensive discussion concerning nonparallel and nonlinear effects on Blasius boundary layers was given by Bertolotti et al. (1992). The underlying notion of the PSE approach is to first decompose the disturbance into an oscillatory wave part and a shape function part. By properly choosing a streamwise wave number to resolve the wave motion, the governing equations reduce to a set of partial differential equations for the shape functions, which vary slowly in the steamwise direction and their second-derivatives are assumed negligible. These PDE's are then parabolized by neglecting the dependence of convected disturbances on downstream events and by neglecting the second derivatives $\left(\partial^{2} / \partial x^{2}\right)$ of the shape functions. Since most of the oscillatory wave motion is absorbed in the streamwise wave number and the terms neglected in the shape function equations are of order $1 / R^{2}$, the resulting system should yield the desired physical results. A brief discussion of the theory is as follows.

For disturbances that are present in the flow field, periodicity is assumed both in time and in the spanwise direction. The total disturbance can then be described by the following Fourier-series expansion:

$$
\{\underline{u}, p\}(x, y, z, t)=\sum_{n=-N_{z}}^{N_{z}} \sum_{m=-N_{t}}^{N_{t}}\left\{\underline{\hat{u}}_{m, n}, \hat{p}_{m, n}\right\}(x, y) \exp [i(n \beta z-m \omega t)]
$$

where $N_{z}$ and $N_{t}$ are the numbers of modes retained in the truncated series, $\omega$ is an imposed frequency, and $\beta$ is an imposed spanwise wave number. Equation (21) is substituted into the governing equations (2) and (3), a set of elliptic equations are obtained for the transformed 
variables $\left\{\underline{\hat{u}}_{m, n}, \hat{p}_{m, n}\right\}$. Because of the wave nature of these transformed variables, a further decomposition is made into a fast-oscillatory wave part and a slow-varying shape function:

$$
\left\{\underline{\hat{u}}_{m, n}, \hat{p}_{m, n}\right\}=\left\{\underline{\tilde{u}}_{m, n}, \tilde{p}_{m, n}\right\} \exp \left[i \int_{x_{o}}^{x} \alpha_{m, n} d \bar{x}\right]
$$

In equation (22), the fast-scale variation along the streamwise direction $x$ is represented by the streamwise wave number $\alpha_{m, n}$; therefore, the second-order variation of the shape function in $x$ is negligible. This observation leads to the parabolized stability equations for the shape functions $\left\{\underline{\tilde{u}}_{m, n}, \tilde{p}_{m, n}\right\}$. These equations are obtained by neglecting all second derivatives in the streamwise direction and the terms associated with upstream influence. If $\alpha_{n, m}$ are chosen properly, the evolution of disturbances can be described by the parabolized equations for the shape functions. Based on decompositions (21) and (22), linear PSE can be derived for any disturbance with a given frequency and spanwise wave number.

To solve the nonlinear problems, the nonlinear convection terms are placed on the right side of the governing equations:

$$
\underline{F}(x, y, z, t)=(\underline{u} \cdot \nabla) \underline{u} .
$$

For the PSE approach, the governing equations are solved in wave number space. The Fourier coefficients, which are obtained from the corresponding Fourier transform of $\underline{F}$ in equation (23), provide a nonlinear forcing to each of the linearized shape-function equations. These inhomogeneous equations for the shape functions are solved by applying a marching procedure along the streamwise direction for each Fourier mode.

Both Bertolotti (1991) and Chang et al. (1991) have extended the PSE numerical approach to the study of compressible boundary layers. The PSE code developed by Chang et al. (1991) with $M_{\infty} \simeq 0$ is used for the present comparison with both the DNS results and the experimental results. A second-order backward differencing is employed to integrate the equations in the streamwise direction, and fourth-order, finite-difference schemes are used to discretize the normal derivatives. 


\section{DNS and PSE Boundary Condition Treatment.}

For the DNS approach, the Navier-Stokes equations with imposed boundary conditions are solved for full disturbance components, which is equivalent to the left side of equation (21). For PSE theory, equations (21) and (22) are substituted into the Navier-Stokes equations (the DNS equations) to produce a coupled system of equations for the unknown Fourier coefficients $\left(\tilde{u}_{m, n}, \tilde{p}_{m, n}\right)$. Boundary conditions are imposed for each Fourier-coefficient equation. Because the PSE theory solves a system of equations in Fourier-coefficient space, more degrees of freedom exist compared with the DNS.

At the wall, no-slip boundary conditions (i.e., homogeneous Dirichlet conditions) are enforced for the disturbance equations used by the DNS and the Fourier-coefficient equations used by PSE theory. So, both DNS and PSE incorporate the same boundary conditions at the wall. In the far field, homogeneous Dirichlet boundary conditions are imposed for the DNS computations. This far-field condition is exact at infinity, but to computationally solve the system using DNS the semi-infinite domain is truncated.

For the PSE approach, homogeneous Dirichlet boundary conditions are used for all Fourier-coefficient equations except for the mean-flow distortion equations. This nonzero component arises from assumptions of PSE theory. Unlike the DNS which solves the full Navier-Stokes equations, PSE theory reduces the equations to a simplified parabolic system in a manner described in the previous subsection. As a result of this PSE simplification, the mean-flow distortion equations become essentially of the boundary-layer equation type. With boundary-layer equations, the wall-normal velocity component approaches a constant in the far-field. Similarly, the mean-flow distortion equation in PSE theory, which is the boundary-layer equation type, incorporates a Neumann boundary condition for the wallnormal velocity component. This Neumann condition allows the total normal velocity (mean flow + mean-flow correction) in the far-field predicted by PSE theory to vary at infinity.

Thus, the far-field boundary conditions used by both the DNS and PSE approaches 
are approximate. The spatial DNS far-field boundary conditions cannot be changed to mimic the PSE approach because the DNS cannot accomodate an a priori Fourier-modal boundary-condition treatment as is present in PSE theory. Changing the Neumann condition to Dirichlet resulted in numerical instabilities in the PSE approach. As a result, the farfield boundary conditions for PSE theory are different from the boundary conditions for the DNS approach for the mean-flow distortion equation. With the present boundary-conditions, the PSE theory approximate far-field conditions should prove more accurate for a far-field boundary fixed close to the wall, and the DNS conditions should prove more realistic for the boundary far from the wall.

\section{Results}

The spatial DNS approach has been tested against linear stability theory for grid and time-step requirements as described by Joslin et al. (1992a). Excellent agreement in the amplitudes and phase of disturbances was found between the DNS results and linear theory; those results will not be repeated in this paper. Rather, three vastly different instability cases have been selected for study: two-dimensional Tollmien-Schlichting wave propagation, subharmonic breakdown, and oblique-wave breakdown. The reasons for the selection of each case will be discussed in each subsection below. These test cases serve to provide a comprehensive evaluation of the effectiveness of PSE theory in predicting convective disturbance development in boundary-layer flows on flat plates.

\section{2-D Tollmien-Schlichting Wave Propagation.}

For this test case, the evolution of an ingested 2-D Tollmien-Schlichting wave is predicted by PSE theory and compared with the DNS results. Because the nonlinear evolution of a single 2-D wave offers a less complex instability mechanism for the boundary-layer problem, this test case is a logical first choice for evaluating PSE theory.

Bertolotti et al. (1992) performed a similar comparison between DNS and PSE theory

for the $u$-velocity components of the fundamental and first harmonic waves. In their work, 
a body-force term was used in the governing equations to introduce the disturbance for the DNS. Then a prescribed inflow (generated by weakly nonlinear theory) was used for disturbance initiation for the PSE. Their results showed good agreement between the fundamental wave and first harmonic amplitudes with downstream distance. If a discrepancy in the results had occurred, one might attribute the difference in results to either a PSE theory flaw or to the different means by-which the disturbances were forced. The intention here is to remove the possibility of variations in disturbance forcing, so the results can be compared without this potential source of confusion. For the present study, the exact same inflow profiles and amplitudes for disturbance forcing are used for both PSE and DNS approaches. As a result, the outcome of the comparison should not depend on a variation of disturbance forcing.

A 2-D Tollmien-Schlichting disturbance with a root-mean-squared (r.m.s.) amplitude $A_{1,0}^{o}=0.0025$ is introduced into the boundary layer by a forcing at the inflow for the DNS as well as for the PSE calculations. (Note: the definition of $A_{m, n}^{o}$ is given in equation (5).) Through nonlinear interactions, all other harmonic waves including the mean-flow distortion are generated for both DNS and PSE. Calculations are made with an inflow Reynolds number $R_{\delta_{0}^{*}}=688.315$ and frequency $F=86$. To generate resolved benchmark data to test PSE theory, the spatial DNS was computed on a grid of 2041 uniformly spaced streamwise nodes (60 nodes per disturbance wavelength) and 81 wall-normal collocation points. The outflow boundary is $442 \delta_{0}^{*}$ from the inflow boundary, and the far-field (or free-stream) boundary is $75 \delta_{0}^{*}$ from the wall. The DNS parameters were chosen based on convergence studies by Joslin et al. (1992a). For the time-marching scheme, the disturbance period is divided into 320 time steps. For the PSE computational approach, several numerical experiments have been performed by varying the grid, far-field boundary location, and the number of Fourier modes. These numerical experiments led to the choice of 100 wall-normal grid points; 5 frequency modes of series (21) $\left(N_{t}=6\right)$; and a far-field boundary located $58 \delta_{o}^{*}$ from the wall.

Figure 1 shows the maximum streamwise amplitudes for the mean-flow distortion $u_{o}$, 
fundamental wave $u_{1}$, and first harmonic $u_{2}$ predicted by PSE theory and compared to the DNS results with the downstream distance. Both the fundamental waves and the first harmonics are in good quantitative agreement throughout the initial linear region and the later weakly nonlinear region. The PSE results capture early evidence of the first harmonic between $R_{\delta^{*}}=690$ and $R_{\delta^{*}}=900$ (barely visible), which is in good agreement with the DNS results. The mean-flow distortion components are in good agreement throughout the initial linear region; however, a discrepancy begins to occur downstream at an apparent "notch" in the results at $R_{\delta^{*}}=1400$. This notch will be explained by looking at the profiles of the meanflow distortion. At the local streamwise location $R_{\delta^{*}}=1519$, figure 2 shows comparisons of the streamwise velocity component. The fundamental (TS) wave and harmonics (not shown) are in good quantitative agreement, even in regions of high gradients. Similar to the discrepancy in the mean-flow distortion amplitudes shown in figure 1, a comparison of the streamwise velocity $\left(u_{o}\right)$ profiles illustrates a comparable difference. Although not shown here, the comparison of the mean-flow distortion at smaller Reynolds numbers gives much better agreement, as expected from figure 1 . The notch in the mean-flow distortion results identified in figure 1 arises from the change of profile contributions. Initially, the positive value of the profile near the wall has a larger amplitude than the retarded, or negative, profile amplitude. At the downstream location near $R_{\delta^{*}}=1400$, the negative portion of the mean-flow distortion becomes the dominant amplitude, and the amplitude of the positive profile near the wall begins to decay. Shown in figure 3, comparisons of the wall-normal velocity $\left(v_{o}\right)$ profiles indicate a larger discrepancy between DNS and PSE theory results. This discrepancy is due to the homogenous Neumann boundary conditions used in the far field for the mean-flow distortion equations in PSE theory. As in the traditional boundarylayer equations approach, this boundary condition leads to a nonzero, wall-normal mean-flow velocity component in the far-field, as discussed in some detail in the previous section. As shown in figure 3, the wall-normal component $v_{o}$ approaches a constant for PSE theory. In 
the present case, the modified far-field normal velocity (i.e., the Blasius solution plus the mean-flow distortion) in PSE theory is shown in figure 4 with the Blasius solution. The maximum difference in the mean flows occurs near the location of the maximum amplitude of the fundamental wave and corresponds to the largest difference in the mean-flow distortion components shown in figure 1.

In this first test problem of 2-D Tollmien-Schlichting wave propagation, the results from DNS and PSE theory agree very well for the fundamental and harmonic waves. However, a discrepancy exists in the mean-flow distortion component. This discrepancy is a result of the difference in the far-field boundary-condition treatment for the two appoaches.

\section{Subharmonic Breakdown.}

A well-understood breakdown scenario in an incompressible boundary layer on a flat plate involves 2-D disturbances and the emergence of aligned and staggered 3-D distinct vortex structures through spanwise vortex stretching and tilting, or some indistinct, nonunique combination of vorticities in the later stages. These vortex patterns are referred to as fundamental, subharmonic, and combination resonant modes, respectively, and may be described by secondary instability theory. The goal here is to use this test case for PSE validation; however, secondary instability theory will not be discussed. For a good review of secondary instabilities, refer to the work by Herbert (1988). For the present study, the subharmonic mode of secondary instability breakdown will be computed and the results compared with the experiments of Kachanov and Levchenko (1984) (hereafter, K\&L). Previously, Fasel et

al. (1990) used spatial DNS and Herbert (1991) used PSE theory and compared their computed subharmonic breakdown results with the K\&L experimental data. The results for both the computation and theory were in relatively good agreement with the experiments. In Herbert's work, comparisons between PSE theory and the spatial DNS results of Fasel et al. (1990) agreed well quantitatively for the modes shown; however, as discussed with the previous 2-D test case, the PSE and DNS approaches used different methods to introduce 
the disturbance. The 3-D disturbances were introduced into the DNS computations by wall suction and blowing while the PSE disturbances were introduced at the inflow.

In the present study, the subharmonic breakdown mechanism is revisited. So that a detailed evaluation of the PSE theory results is possible, both DNS and PSE theory must use the same initial disturbance amplitudes and profiles, which are obtained from linear and secondary instability theory. Furthermore, physical features of the K\&L experiments differed from the earlier computations by both Fasel and Herbert with respect to some of the modal trends. In the present paper, an explanation for these discrepancies will be sought.

For spatial DNS, computations are performed on a grid of 1021 uniformly spaced streamwise nodes, 81 wall-normal collocation points, and 5 symmetric-spanwise nodes. In the streamwise direction, the outflow boundary is $442 \delta_{0}^{*}$ from the inflow boundary; the far field boundary is $75 \delta_{o}^{*}$ from the wall; and the spanwise boundary consists of a length equal to one half of the spanwise wavelength, or $\lambda_{z} / 2=\pi / \beta$. (Note that the spanwise computational length would be $\lambda_{z}$ for the general, nonsymmetric computation.) For the time-marching scheme, the disturbance period is divided into 320 time steps, and time is advanced using a three-stage Runge-Kutta method. For the PSE computational approach, 100 wall-normal grid points are used; 7 frequency modes and 3 spanwise modes from series (21) are used, and the far-field boundary is $58 \delta_{0}^{*}$ from the wall.

The prescribed primary and subharmonic disturbances are obtained at the Reynolds number $R_{\delta_{0}^{*}}=732.711$ and the primary frequency $F=124$ (which correspond to the experiments of K\&L). The primary wave has an inflow amplitude of $A_{2,0}^{o}=0.0048$ (r.m.s.). The subharmonic mode has an inflow amplitude of $A_{1,1}^{o}=0.145 \times 10^{-4}$ (r.m.s.) and corresponds to a mode with spanwise wave number $\beta=0.2418$.

Figure 5 compares the maximum amplitudes predicted by PSE theory with the results from DNS. For this test case, note the extremely good quantitative agreement between PSE theory and DNS for the growth rates of the fundamental and the dominant harmonic modes; 
even the mean-flow distortion components are in good agreement. PSE theory and DNS profiles are compared in figure 6 at the local (downstream) Reynolds number $R_{\delta^{*}}=1067$. The predictions made by PSE theory are in good overall quantitative agreement with DNS results. From these comparisons, the accuracy of the PSE predictions has been established for this test case.

The second goal is to understand the modal discrepancies identified from the comparison with the experiments. In figure 7 , the DNS results are compared with results of the K\&L experiments. Furthermore, the computed profiles are in good agreement with the K\&L experiments as shown in figure 8 at the downstream location where $R_{\delta^{*}}=1049$. Both the earlier DNS results of Fasel et al. (1990) and PSE results of Herbert (1991) indicated good agreement with the K\&L experiments, similar to the present results. But, if the results are examined more carefully on a mode-by-mode basis, modal inconsistencies can be found and lead to distinct differences in the interpretation of the computational and experimental results. In particular, the primary wave $(F)$ and its harmonics $(2 F, 3 F)$ begin to decay much sooner downstream in the experiments $\left(R_{\delta^{*}} \simeq 990\right)$ than in the computations $\left(R_{\delta^{*}} \simeq 1070\right)$. This observation can be seen clearly in figure 9 ; the fundamental waves of DNS and the experiments are shown with downstream development. A reason for this difference will be briefly explored.

Based on linear stability theory, the discrepancy in the 2-D fundamental mode (shown in figure 9) can be attributed to the fact that each wave may have a different frequency. The fundamental wave in the experiments reaches a peak amplitude and then decays upstream of the theoretical wave, which suggests that the experiment has a larger effective frequency than in the computations and the theoretical predictions. Furthermore, K\&L note that a pressure gradient of no more than a 0.8 percent per one meter may have been present in the experiments. From linear theory, stability results are known to be very sensitive to pressure gradients. If a small, unmeasurable adverse pressure gradient was present, then the 
fundamental wave would reach larger amplitude levels, as recorded. Individually, the effects of pressure gradient and frequency differences can not explain the differences between the computational and experimental results. So to determine the combined effect of frequency and pressure-gradient variations on the fundamental wave $F$ and harmonics $2 F, 3 F$, PSE theory is used to re-compute the subharmonic instability case.

Using linear stability theory as a guide, a frequency $F=140$ and a small adverse pressure gradient $\beta_{h}=-0.02$ are selected for another computation using PSE theory. The pressure gradient parameter $\beta_{h}=-0.02$ which corresponds to $d p / d x=0.9$ percent per unit length is of the same order as the possible error given in the experiments. Figures 10 and 11 compare these new results with the previous DNS results and experiments. As shown in figure 10, with this modification of the frequency and base flow, the growth and decay rates of the fundamental wave are in much better agreement with the experiments downstream of $R_{\delta^{*}}=900$. The growth rates prior to $R_{\delta^{*}}=900$ amplify different frequencies in the experiments and computations. In figure 11, the computed primary mode $(F)$ and higher modes $(2 F, 3 F)$ more closely follow the trend toward decay exhibited in the experiments; however, the growth rates of the subharmonic-based modes $(F / 2$ and $3 F / 2)$ have increased notably as a result of the higher frequency. The earlier PSE and DNS results more closely match the subharmonic and its harmonics in the experiments. Although it is unlikely that such a large frequency discrepancy from $F=124$ noted in the experiments occurred, it is clear that the available experimental data is not sufficient to reproduce the results numerically.

\section{Oblique-Wave Breakdown.}

The final test case used in the evaluation of PSE theory is oblique-wave breakdown. This oblique-wave breakdown procedure is due to the nonlinear interactions of a pair of oblique waves. Because of this nonlinearity, no adequate formal theory is available to explain the breakdown process; however, similar mechanisms have been studied by Hall and Smith (1991) with asymptotic methods. Hall and Smith discussed the vortex-wave interactions within a 
large wave number and Reynolds number limit. To quantify the mechanisms of interest in the finite Reynolds number range, DNS and possibly PSE theory are the only options available to study the wave interactions.

Because this alternative route to, or mechanism of, transition is nonlinear, limited research has been done for oblique-wave breakdown. It is worth mentioning a few of the interesting papers that are available. Schmid and Henningson (1992a) studied bypass transition by introducing a pair of large-amplitude oblique waves into channel flow. The evolution of disturbances was computed with temporal DNS. They found that the development of the oblique waves was dominated by a preferred spreading of the energy spectra into low streamwise wave numbers, which led to the rapid development of streamwise-elongated structures. Schmid and Henningson (1992b) also looked at small-amplitude wave pairs over a variety of parameters. They suggest that the mechanism of energy transfer is primarily linear. Fasel (1991), Fasel and Thumm (1991), and Bestek et al. (1992) computed this new breakdown structure in a compressible boundary layer. They describe the physical structure as "honeycomb-like" to identify a distinction from the secondary instability $\Lambda$-like structures. Chang and Malik (1992) used PSE theory to examine the breakdown of supersonic boundary layers because the dominant first mode is an oblique wave in supersonic flows. Chang and Malik found that even waves with amplitudes as small as 0.001 percent that are initiated at the lower branch can lead to transition in this breakdown scenario, depending on the frequency of the induced oblique waves. Finally, Joslin et al. (1992b) presented the preliminary results (computed by both spatial DNS and PSE theory) of oblique-wave breakdown in incompressible boundary layers. This paper presents a more comprehensive discussion of oblique-wave breakdown in incompressible boundary layers, and PSE theory is again evaluated for accuracy in predicting this nonlinear flow development.

The profiles for the oblique-wave pair are obtained from linear stability theory for the Reynolds number $R_{\delta_{0}^{*}}=900$, a frequency $\omega=0.0774$, and spanwise wave numbers $\beta= \pm 0.2$. 
Spatial DNS computations are performed on a grid of 901 uniformly spaced streamwise nodes, 61 wall-normal collocation points, and 10 symmetric-spanwise modes. In the streamwise direction, the outflow boundary is $465 \delta_{0}^{*}$ from the inflow boundary; the far-field, or freestream, boundary is $75 \delta_{0}^{*}$ from the wall; and the spanwise boundary consists of a length equal to one half of the spanwise wavelength, or $\lambda_{z} / 2=\pi / \beta$. For the time-marching scheme, the disturbance period is divided into 320 time steps. For the PSE computational approach, 100 wall-normal grid points are used; 7 frequency modes and 7 spanwise modes of series (21) are used; and the far-field boundary is $58 \delta_{0}^{*}$ from the wall.

The input modes are represented by equation (5), which are truncated to four terms. The disturbance forcing consists of modes $(1,1)$ and $(1,-1)$, or $(\omega, \beta)$ and $(\omega,-\beta)$, and their complex conjugates $(-1,1)$ and $(-1,-1)$. Theoretically, if these modes self-interact initially, then only certain higher modes are likely to be excited (supplied energy). These higher modes are: $(0,0),(0,2),(2,0)$, and $(2,2)$, etc.

In the first test case, the oblique waves each have the small amplitude $A_{1,1}^{o}=0.001$. In figure 12 , the primary disturbance $(1,1)$ and the higher modes that were predicted by PSE theory are compared to the DNS results. The comparison shows that the modes are in quantitative agreement. Of the modes that were likely to be excited, all received energy initially. The streamwise vorticity component $(0,2)$ grows rapidly because of the self-interaction of the oblique-wave pair. All other modes grow more slowly downstream than the streamwise vortex, and these other modes contain less energy by orders of magnitude. As a result of the rapid growth of the vortex mode $(0,2)$, the oblique waves interact with the vortex which leads to an amplified harmonic $(1,3)$. This $(1,3)$ mode gains energy sufficient to overtake the other initially excited modes, but insufficient to overtake the oblique waves. As shown in figure 12, the vortex modes self-interact to supply energy to the $(0,4)$ mode, which has roughly the same growth rate as the $(0,2)$ mode. Although the computations were discontinued, the disturbances will eventually decay and will not lead to transition because the 
primary oblique waves decay after they pass the upper branch of the neutral curve, and all other modes are decaying or becoming neutrally stable. Similar to the previous test cases, PSE theory profiles are compared with the spatial DNS for accuracy. In figure 13, streamwise disturbance velocity profiles are shown for the downstream location $R_{\delta^{*}}=1178$. PSE theory predictions agree very well with the DNS results for all modes shown. Note, PSE theory captures the near-wall structure evident in the fundamental wave profile in figure 13 , in agreement with the DNS results.

For a final computation, the oblique waves are introduced with larger amplitudes $A_{1,1}^{o}=$ 0.01. The computed primary disturbance $(1,1)$ and higher modes are shown in figure 14 . Again, the modes predicted by PSE theory are shown to be in agreement with the DNS results. Similar to the small amplitude case, the small wave number modes gain initial energy. The vortex mode $(0,2)$ is again the dominant, higher order mode. The self-interaction of the wave pairs and the interaction with the streamwise vortex lead to a rapid cascade of energy to the other modes. Rather than the meager growth and downstream decay, these higher modes now grow with growth-rate characteristics similar to the vortex mode. The vortex and harmonics rapidly overtake the introduced waves $(1,1)$ and breakdown occurs. At breakdown, the spectrum undergoes a filling so both the DNS and PSE computations are underresolved near the downstream end of figure 14. Further evidence that the onset of transition from laminar to turbulent flow has begun, the skin-friction curve begins to rise. Finally, a comparison of local disturance profiles yields the good agreement between PSE and DNS comparable to the low amplitude comparison of figure 13. 


\section{Concluding Remarks}

In this paper, PSE theory results were evaluated for accuracy in predicting convective disturbance evolution on a flat plate. PSE theory predictions were compared with spatial DNS results for 2-D Tollmien-Schlichting wave propagation, subharmonic breakdown, and oblique-wave breakdown.

For 2-D Tollmien-Schlichting wave propagation, the modes predicted by PSE theory were in very good quantitative agreement with the DNS results, except a small discrepancy in the mean-flow distortion component was discovered and attributed to far-field boundarycondition differences.

For the test case of subharmonic breakdown, the PSE theory results were in very good quantitative agreement with the DNS results for all modes, even the mean-flow distortion component. Also the present study supports the PSE and DNS comparison made by Herbert (1991) for subharmonic breakdown.

The present computations were in good qualitative agreement with the experiments of Kachanov and Levchenko (1984). In light of some modal discrepancies between computations and experiments, a possible explanation was given. By introducing a small, adverse pressure gradient in the basic flow and allowing for a small effective frequency variation in the disturbance, the computations predicted modal trends which were similar to the experiments for the fundamental disturbance.

For the complicated test case of oblique-wave breakdown, all modes predicted by PSE theory were shown to be in good quantitative agreement with the DNS results, even for the mean-flow distortion component. Furthermore, these oblique-wave pairs were shown to selfinteract to excite a streamwise vortex structure, which agrees with the findings of Schmid and Henningson (1992a, 1992b). If the initial wave amplitudes are above a threshhold, the interaction of these waves and the vortex can lead to a breakdown that bypasses the secondary instability stage. Irrespective of the initial amplitudes, the streamwise vortex 
mode becomes the dominant, higher-order mode. This dominance is significant because the presence of small roughness elements may generate oblique wave packets that can interact and lead to the increased presence of streamwise vorticity.

Relevant to the applied engineering community, is the fact that PSE theory has accurately predicted the disturbance development for 2-D Tollmien-Schlichting wave propogation, subharmonic breakdown, and oblique-wave breakdown on a flat plate. A difference in the far-field boundary-condition treatment for the PSE method was identified which may cause mean-flow distortion variations; however, all other components appear to be affected very little as a result of this far-field treatment. In the near future, PSE theory will be a useful tool for the engineer, and for certain types of flow problems, the use of PSE theory is expected to increase. However, because of the assumptions that underlie PSE theory, its application to problems other than those for which it has been adequately tested must be done with caution. For example, can PSE theory handle the potentially absolute instability of a recirculation bubble? Studies of the kind presented in this paper are necessary to provide verification of new theories and to study the unknown complex physics of various flows.

\section{Acknowledgments}

The authors wish to express their gratitude to Dr. Bart A. Singer, High Technology Corporation, for reviewing this manuscript and for providing useful comments to enhance the final draft. Also, thanks goes to Ms. Jonay A. Campbell, Mason and Hanger Services Incorporated, for her editorial assistance that ultimately has led to a more coherent and readable paper.

\section{References}

Benney, D.J., and Lin, C.-C. (1960). On the secondary motion induced by oscillations in a shear flow. Phys. Fluids, 3, 656-657.

Bertolotti, F.P. (1991). Linear and nonlinear stability of boundary layers with streamwise varying properties. Ph.D. Thesis, The Ohio State University. 
Bertolotti, F.P., Herbert, Th., and Spalart, P.R. (1992). Linear and nonlinear stability of the Blasius boundary layer. J. Fluid Mech., 242, 441-474.

Bestek, H., Thumm, A., and Fasel, H.F. (1992). Numerical investigation of later stages of transition in transonic boundary layers. In First European Forum on Laminar Flow Technology, March 16-18, 1992. Hamburg, Germany.

Boyd, J.P. (1989). Chebyshev-Fourier Spectral Methods. Lecture Notes in Physics, 49, Springer-Verlag, New York.

Canuto, C., Hussaini, M.Y., Quarteroni, A., and Zang, T.A. (1988). Spectral Methods $\underline{\text { in }}$ Fluid Dynamics, Springer-Verlag, New York.

Chang, C.-L., Malik, M.R., Erlebacher, G., and Hussaini, M.Y. (1991). Compressible stability of growing boundary layers using parabolized stability equations. AIAA Paper No. $91-1636$.

Chang, C.-L., and Malik, M.R. (1992). Oblique mode breakdown in a supersonic boundary layer using nonlinear PSE. In Instability, Transition, and Turbulence, (M.Y. Hussaini, A. Kumar, and C.L. Streett, eds.), pp. 231-241. Springer-Verlag, New York.

Craik, A.D.D. (1971). Nonlinear resonant instability in boundary layers. J. Fluid Mech., 50, 393-413.

Danabasoglu, G., Biringen, S., and Streett, C.L. (1990). Numerical simulation of spatiallyevolving instability control in plane channel flow. AIA A Paper No. 90-1530.

Danabasoglu, G., Biringen, S., and Streett, C.L. (1991). Spatial simulation of instability control by periodic suction blowing. Phys. Fluids A, 3(9), 2138-2147.

Fasel, H.F. (1976). Investigation of the stability of boundary layers by a finite-difference model of the Navier-Stokes equations. J. Fluid Mech., 78, 355-383. 
Fasel, H.F., Rist, U., and Konzelmann, U. (1990). Numerical investigation of the threedimensional development in boundary-layer transition. AIAA J., 28(1), 29-37.

Fasel, H.F. (1991). (private communication).

Fasel, H.F., and Thumm, A. (1991). Numerical simulation of three-dimensional boundarylayer transition. Bull. Am. Phys. Soc., 36, 2701.

Grosch, C.E., and Orszag, S.A. (1977). Numerical solution of problems in unbounded regions: coordinate transforms. J. Comput. Phys., 25, 273-296.

Hall, P., and Smith, F.T. (1991). On strongly nonlinear vortex/wave interactions in boundary-layer transition. J. Fluid Mech., 227, 641-666.

Herbert, Th., and Bertolotti, F.P. (1987). Stability analysis of nonparallel boundary layers. Bull. Am. Phys. Soc., 32, 2079.

Herbert, Th. (1984). Secondary instability of shear flows. AGARD-R-709.

Herbert, Th. (1988). Secondary instability of boundary layers. Ann. Rev. Fluid Mech., 20, $487-526$.

Herbert, Th. (1991). Boundary-layer transition-analysis and prediction revisited. AIAA Paper No. 91-079\%.

Joslin, R.D., Streett, C.L., and Chang, C.-L. (1992a). Validation of three-dimensional incompressible spatial direct numerical simulation code-a comparison with linear stability and parabolic stability equations theories for boundary-layer transition on a flat plate. NASA TP-3205.

Joslin, R.D., Streett, C.L., and Chang, C.-L. (1992b). Oblique-wave breakdown in an incompressible boundary layer computed by spatial DNS and PSE theory. In Instability, Transition, and Turbulence, (M.Y. Hussaini, A. Kumar, and C.L. Streett, eds.), pp. 304-310. Springer-Verlag, New York. 
Kachanov, Y.S., Kozlov, V.V., and Levchenko, V.Y. (1977). Nonlinear development of a wave in a boundary layer. Izv. Akad. Nauk SSSR, Mekh. Zhidk. i Gaza, 3, 49-56 (in Russian). (Translation: Fluid Dynamics, 12, 1978, 383-390.)

Kachanov, Y.S., and Levchenko, V.Y. (1984). The resonant interaction of disturbances at laminar-turbulent transition in a boundary layer. J. Fluid Mech., 138, 209-247.

Klebanoff, P.S., and Tidstrom, K.D. (1959). Evolution of amplified waves leading to transition in a boundary layer with zero pressure gradient. NACA TN D-195.

Klebanoff, P.S., Tidstrom, K.D., and Sargent, M.L. (1962). The three-dimensional nature of boundary-layer instability. J. Fluid Mech., 12, 1-34.

Kleiser, L., and Zang, T.A. (1991). Numerical simulation of transition in wall-bounded shear flows. Ann. Rev. Fluid Mech., 23, 495-537.

Laurien, E., and Kleiser, L. (1989). Numerical simulation of boundary-layer transition and transition control. J. Fluid Mech., 199, 403-440.

Lele, S.K. (1992). Compact finite difference schemes with spectral-like resolution. J. Comput. Phys., 103, 16-42.

Lynch, R.E., Rice, J.R., and Thomas, D.H. (1964). Direct solution of partial difference equations by tensor product methods. Num. Math., 6, 185-199.

Orr, W.M.A. (1907). The stability or instability of the steady motions of a perfect liquid and of a viscous liquid. Roy. Irish Acad., 27, 9-138.

Orszag, S.A., and Patera, A.T. (1980). Subcritical transition to turbulence in plane shear flows. Phys. Rev. Lett., 45, 989-993.

Orszag, S.A., and Patera, A.T. (1983). Secondary instability of wall-bounded shear flows. J. Fluid Mech., 128, 347-385. 
Rai, M.M., and Moin, P. (1991a). Direct numerical simulation of transition and turbulence in a spatially-evolving boundary layer. AIAA Paper No. 91-160\%.

Rai, M.M., and Moin, P. (1991b). Direct numerical simulation of turbulent flow using finitedifference schemes. J. Comput. Phys., 96, 15-53.

Schlichting, H. (1933). Zur die enstehung der turbulenz bei der plattenströmung. Nachrichten von der Gesellschaft der Wissenschaften zu Gottingen, MathematischPhysikalische Klasse, 181-208.

Schlichting, H. (1955). Boundary-Layer Theory. McGraw-Hill, New York.

Schmid, P.J., and Henningson, D.S. (1992a). Channel flow transition induced by a pair of oblique waves. In Instability, Transition, and Turbulence, (M.Y. Hussaini, A. Kumar, and C.L. Streett, eds.), pp. 356-366. Springer-Verlag, New York.

Schmid, P.J., and Henningson, D.S. (1992b). A new mechanism for rapid transition involving a pair of oblique waves. Phys. Fluids A, 4(9), 1986-1989.

Schubauer, G.B., and Skramstad, H.K. (1943). Laminar boundary-layer oscillations and transition on a flat plate. NACA TR-909, (Originally NACA ACR, April 1943).

Schubauer, G.B., and Skramstad, H.K. (1947). Laminar boundary-layer oscillations and stability of laminar flow. J. Aeronaut. Sci., 14(2), 69-78.

Smith, A.M.O., and Gamberoni, N. (1956). Transition, pressure gradients, and stability theory. Douglas Aircraft Company Report No. ES-26388.

Smith, F.T. (1979). Nonlinear stability of boundary layers for disturbances of various sizes. Proc. Roy. Soc. Lond. A, 368, 573-589.

Sommerfeld, A. (1908). Ein beitrag zur hydrodynamischen erklaerung ler turbulenten flüessigkeitsbewegunger. Int. Congr. Math., 116-124. 
Spalart, P.R. (1989). Direct numerical study of leading-edge contamination. In Fluid Dynamics of Three-Dimensional Turbulent Shear Flows and Transition, AGARD-CP-438, 5.1-5.13. Streett, C.L., and Macaraeg, M.G. (1989). Spectral multi-domain for large-scale fluid dynamic simulations. Int. J. Appl. Numer. Math., 6, 123-140.

Streett, C.L., and Hussaini, M.Y. (1991). A numerical simulation of the appearance of chaos in finite-length Taylor-Couette flow. Appl. Numer. Math., 7, 41-71.

Tollmien, W. (1929). Über die entstehung der turbulenz. Nachrichten von der Gesellschaft der Wissenschaften zu Gottingen, Mathematisch-Physikalische Klasse, 21-44. (translated in NACA TM-609, 1931).

Van Ingen, J.L. (1956). A suggested semi-empirical method for the calculation of the boundary-layer transition region. University of Delft, Department of Aerospace Engineering, Delft, The Netherlands Report VTH-74.

Williamson, J.H. (1980). Low-storage Runge-Kutta schemes. J. Comput. Phys., 35(1), $48-56$.

Zang, T.A., and Hussaini, M.Y. (1987). Numerical simulation of nonlinear interactions in channel and boundary-layer transition. Nonlinear Wave Interactions in Fluids, 87, 131-145. Zang, T.A., and Hussaini, M.Y. (1990). Multiple paths to subharmonic laminar breakdown in a boundary layer. Phys. Rev. Lett., 64, 641-644. 

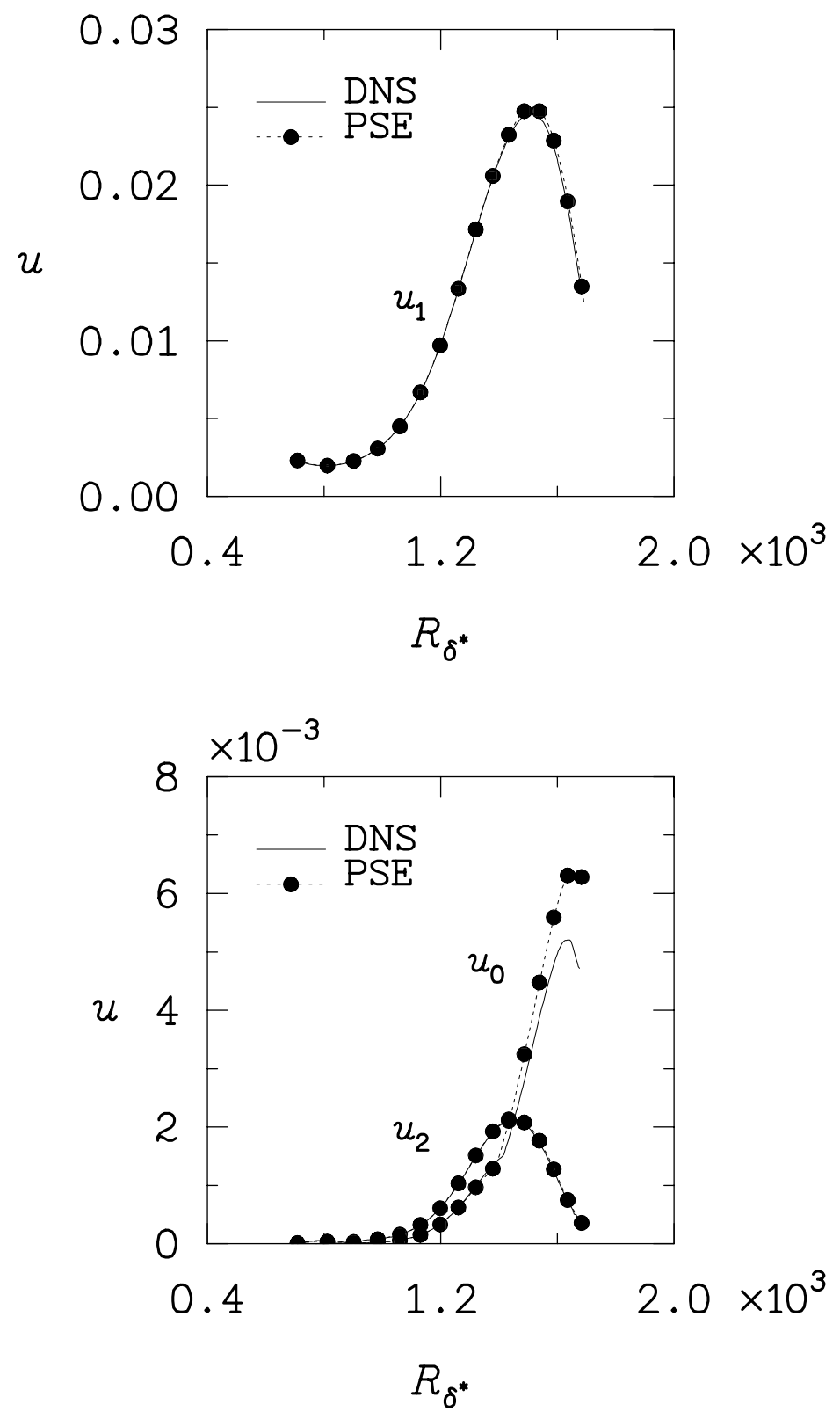

Figure 1. Amplitude growth with downstream distance, from two-dimensional wave with amplitude $A_{1,0}^{o}=0.0025$ (r.m.s.). 

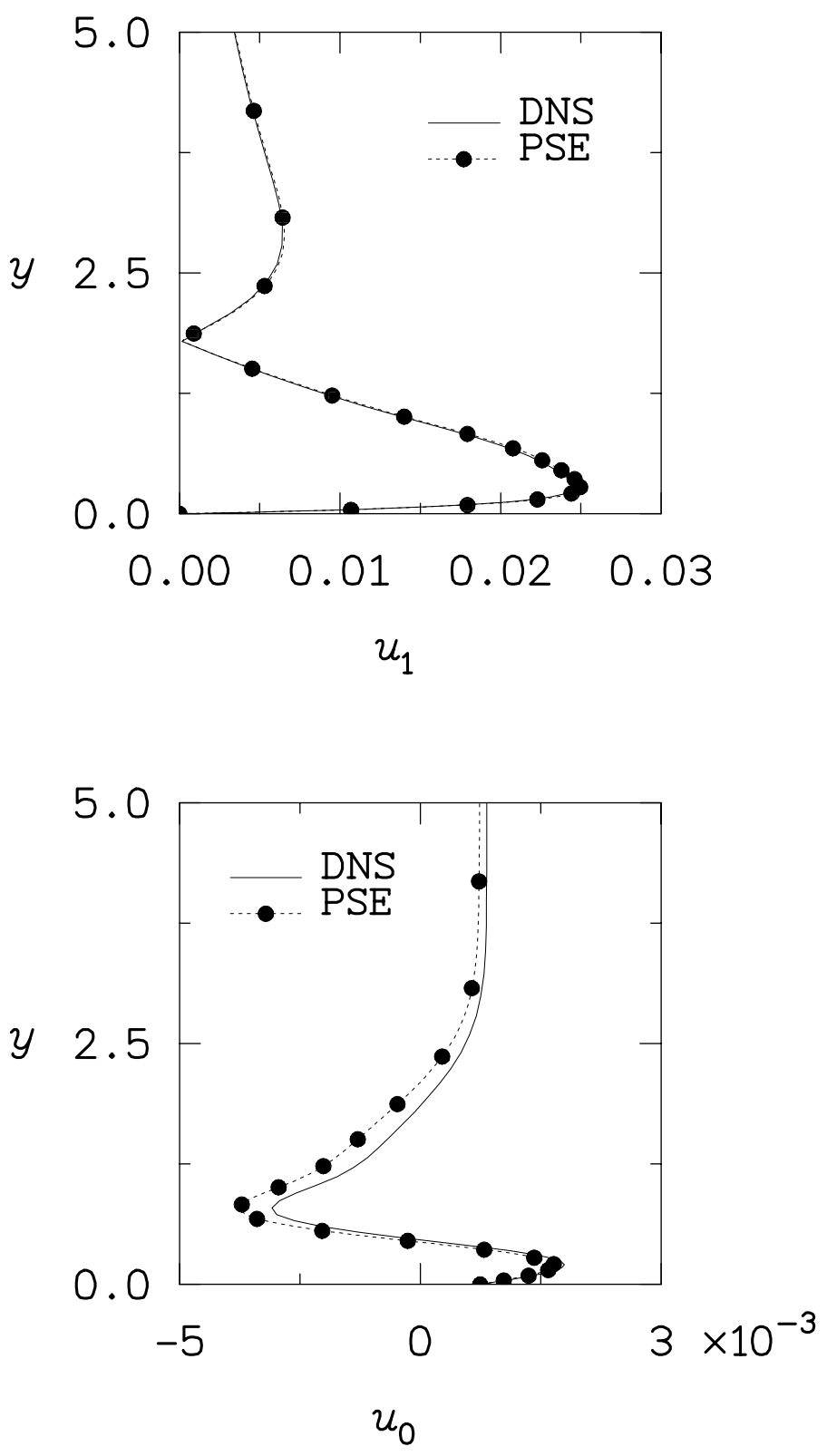

Figure 2. Streamwise $(u)$ mean-flow distortion and fundamental wave profiles with normal distance from the wall at the local Reynolds number $R_{\delta^{*}}=1519$. 

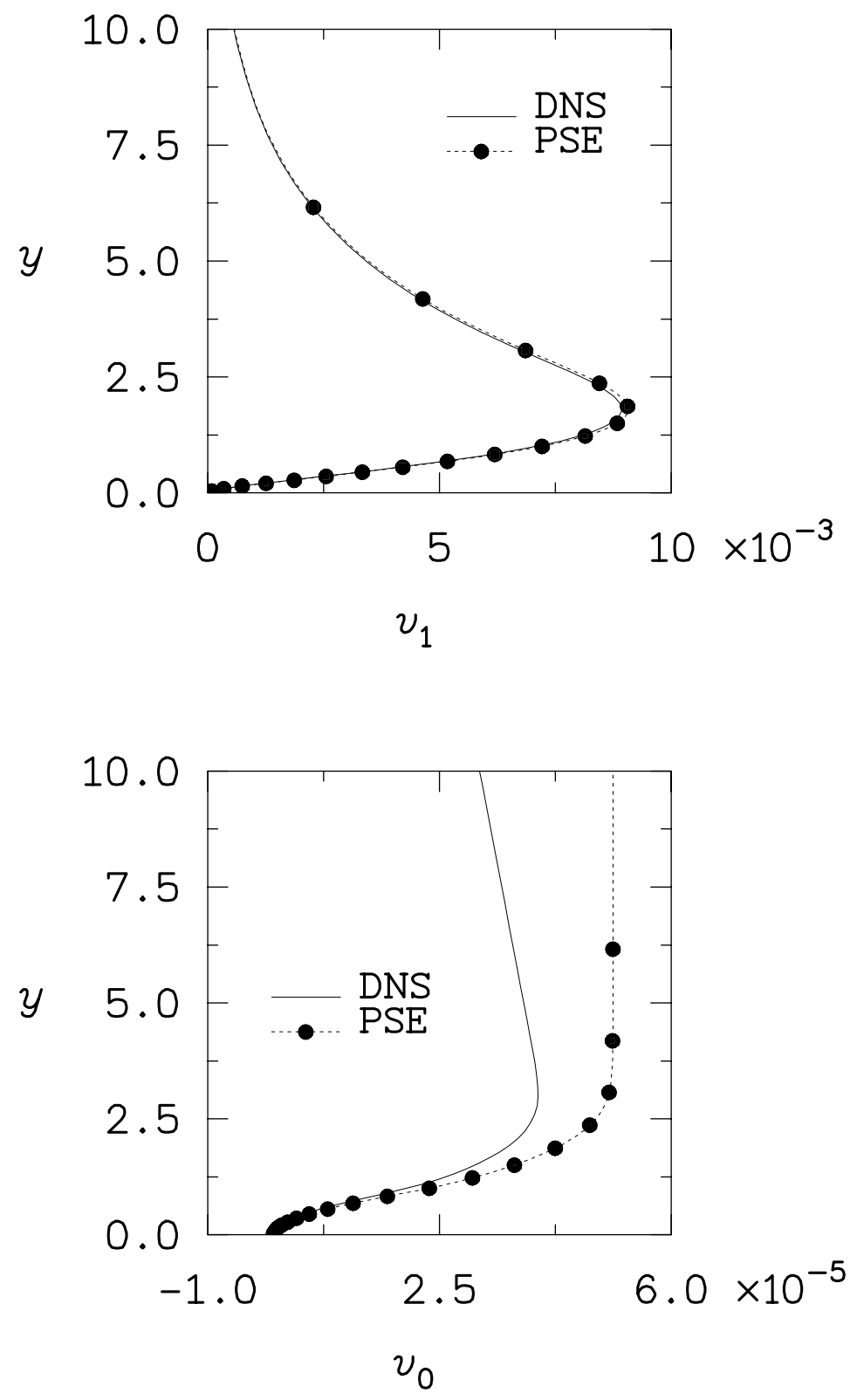

Figure 3. Normal $(v)$ mean-flow distortion and fundamental wave profiles with normal distance from the wall at the local Reynolds number $R_{\delta^{*}}=1519$. 


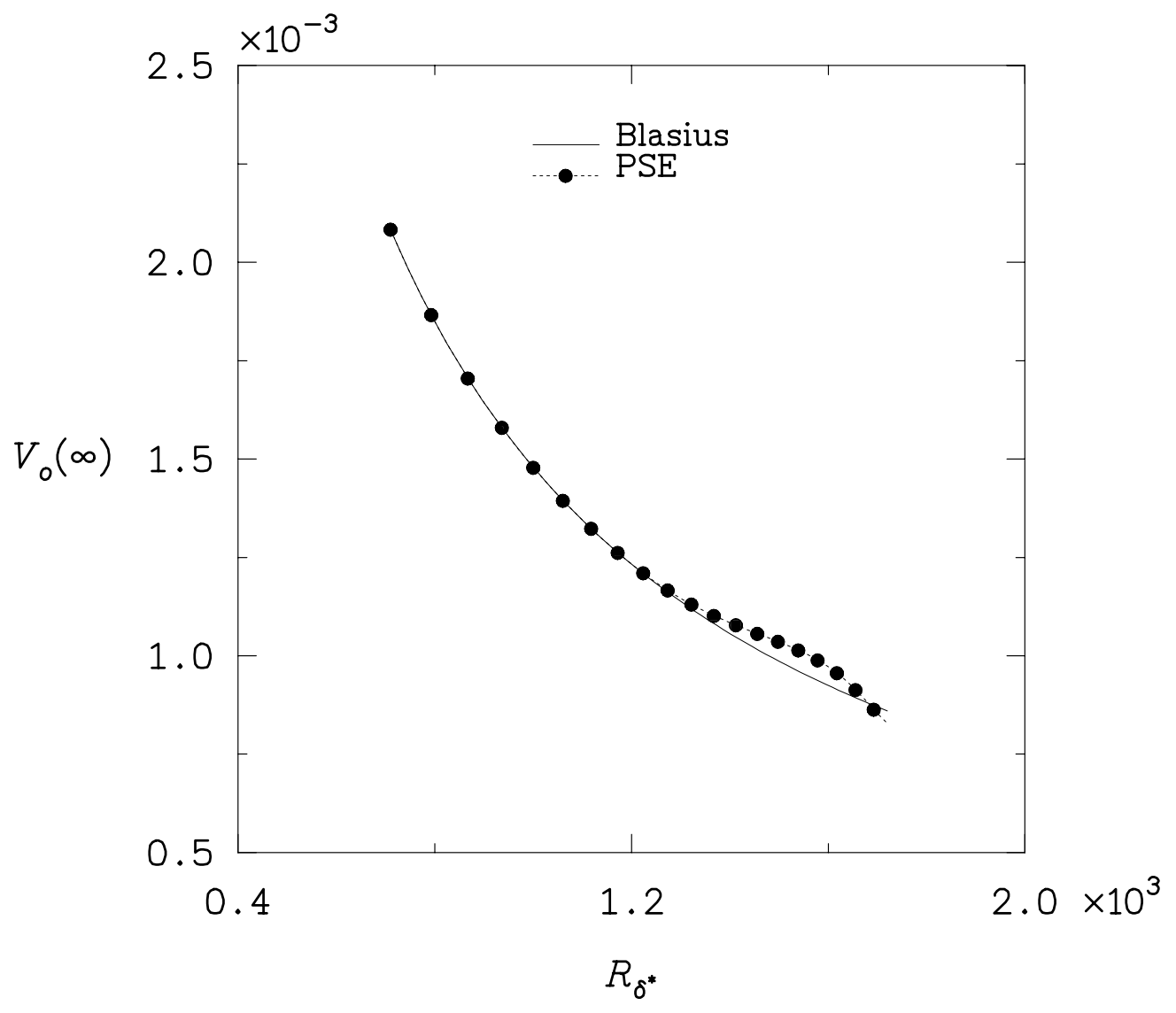

Figure 4. Normal component of the mean flow $\left(V_{o}\right)$ at the far-field boundary. 


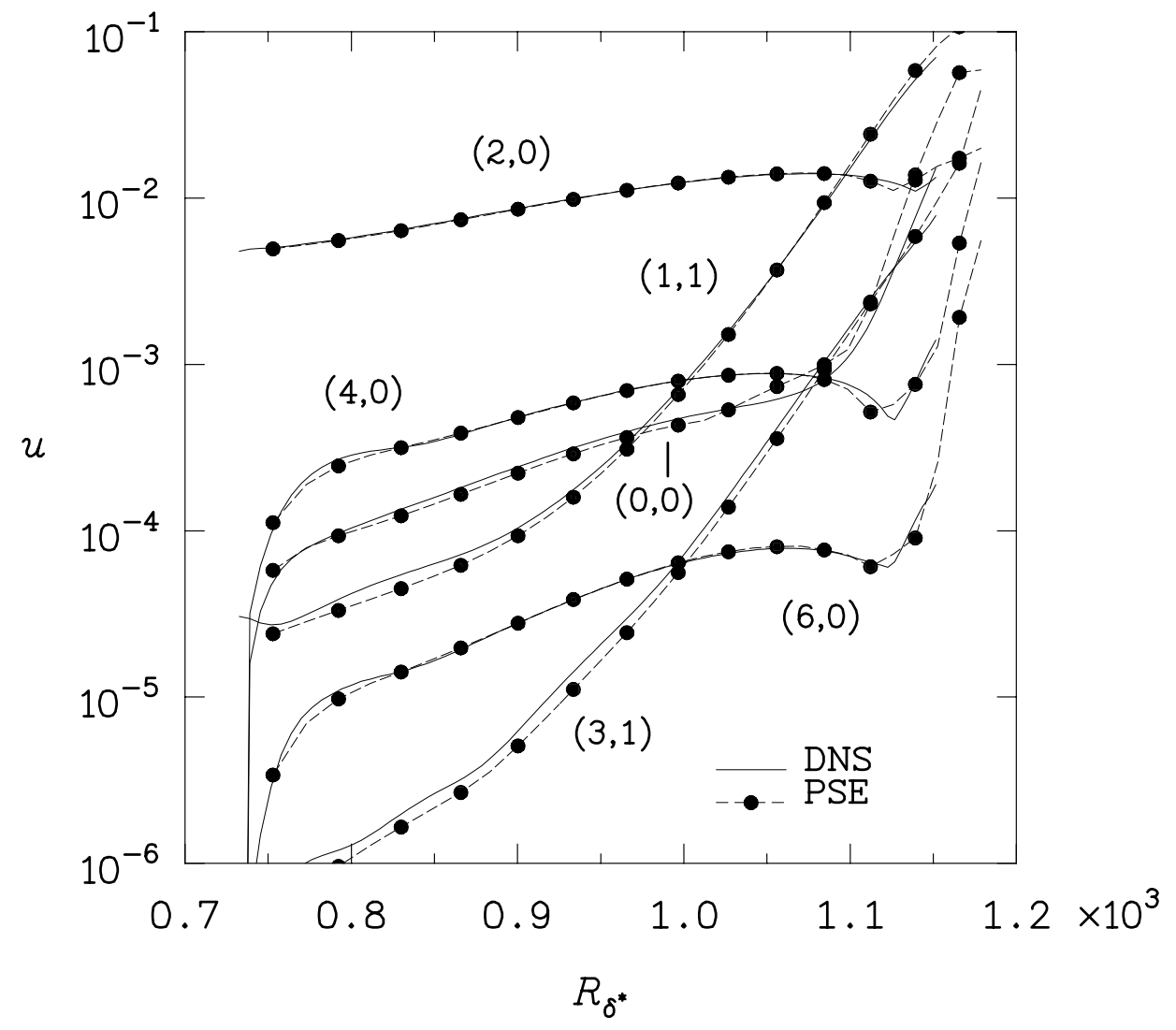

Figure 5. Maximum amplitude growth with downstream distance for the primary wave $[F=(2,0)]$, subharmonic $[F / 2=(1,1)]$, and various higher harmonics $[2 F=(4,0), 3 F=$ $(6,0), 3 F / 2=(3,1)]$. 

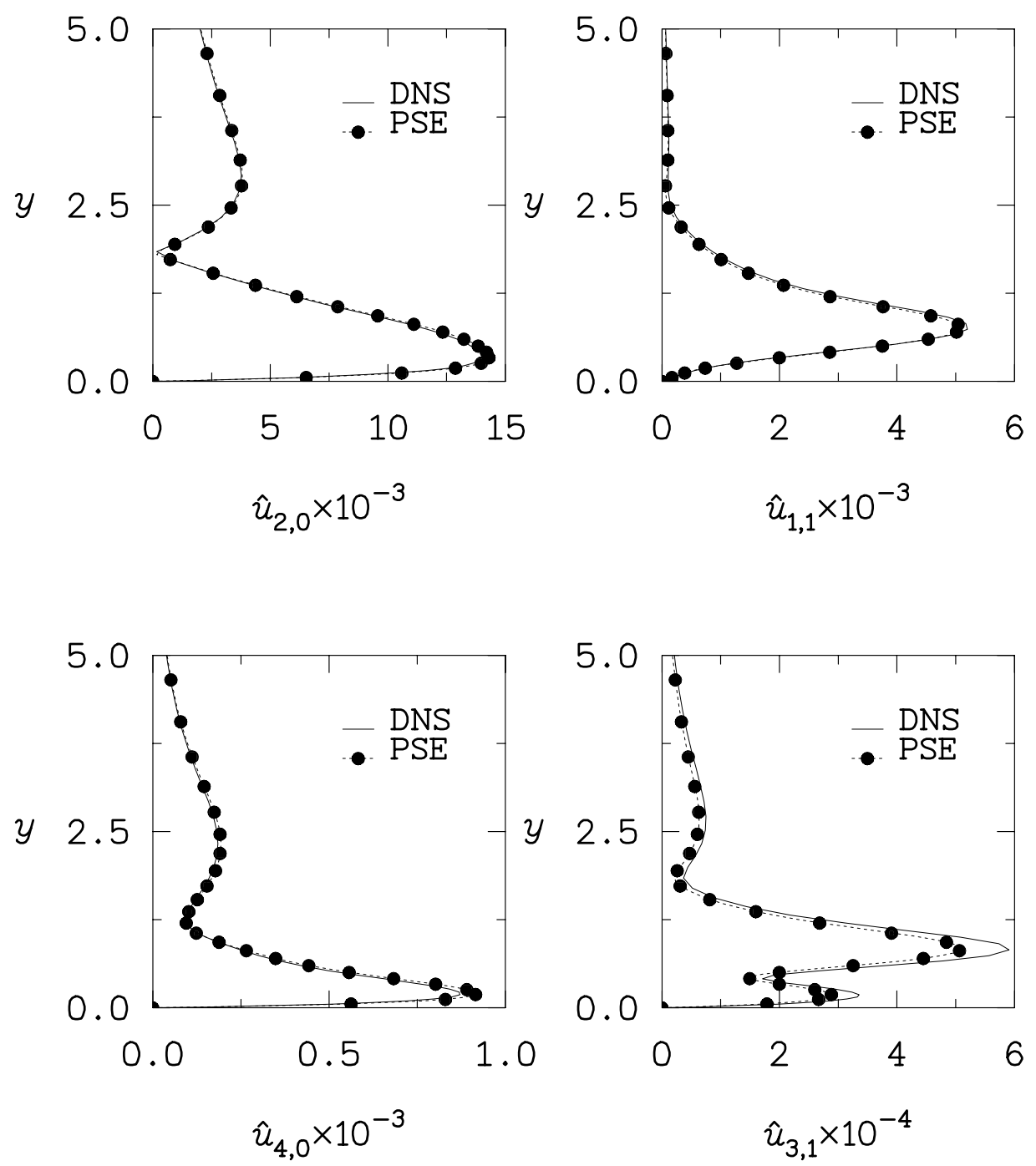

Figure 6. Streamwise disturbance profiles at the downstream location where locally $R_{\delta^{*}}=$ 1067 for subharmonic breakdown. $[F=(2,0), 2 F=(4,0), F / 2=(1,1), 3 F / 2=(3,1)]$ 


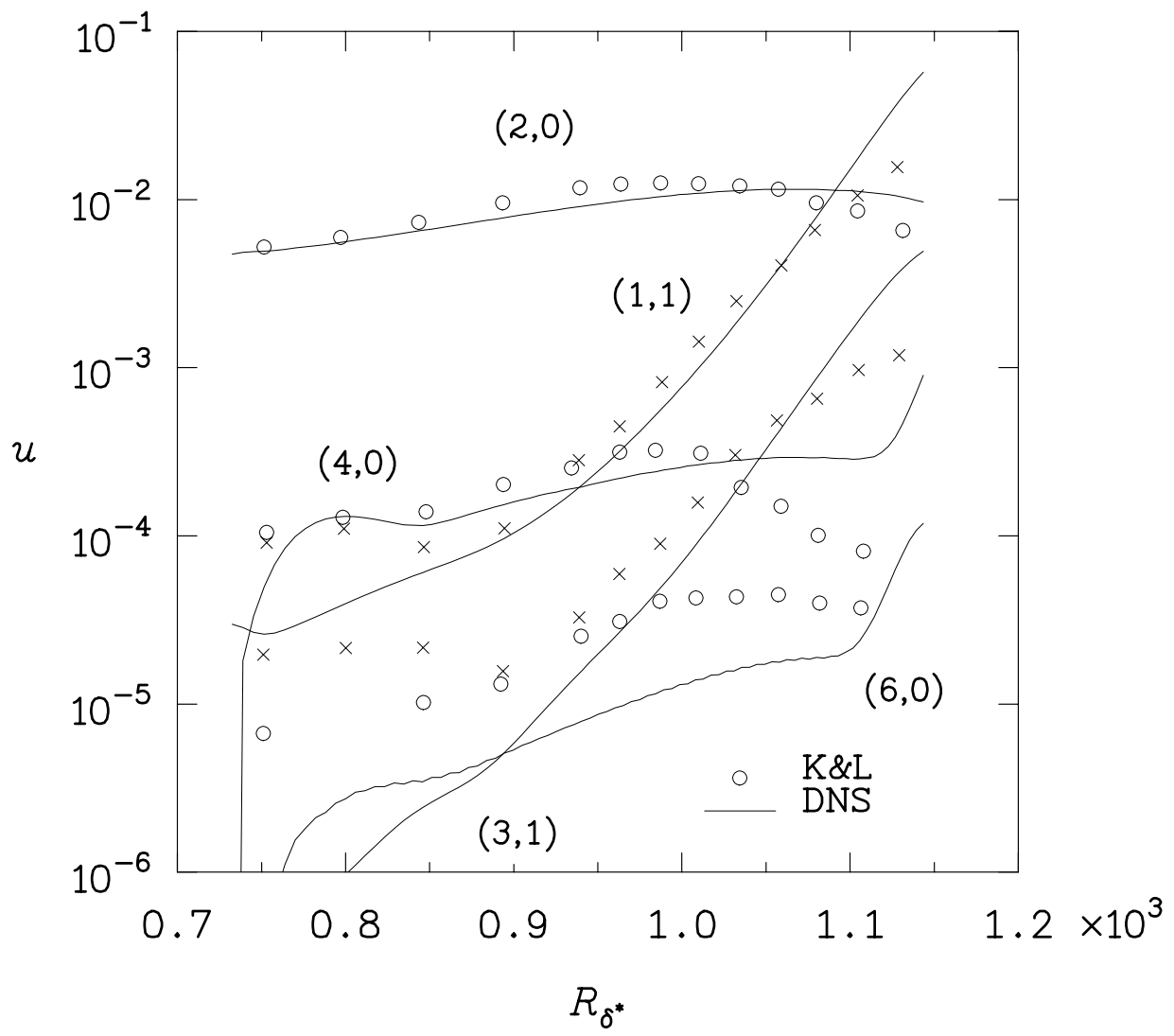

Figure 7. Amplitude growth with downstream distance for the primary wave $[F=(2,0)]$, subharmonic $[F / 2=(1,1)]$, and various higher harmonics $[2 F=(4,0), 3 F=(6,0), 3 F / 2=$ $(3,1)]$ at $y=0.26 \delta$. 

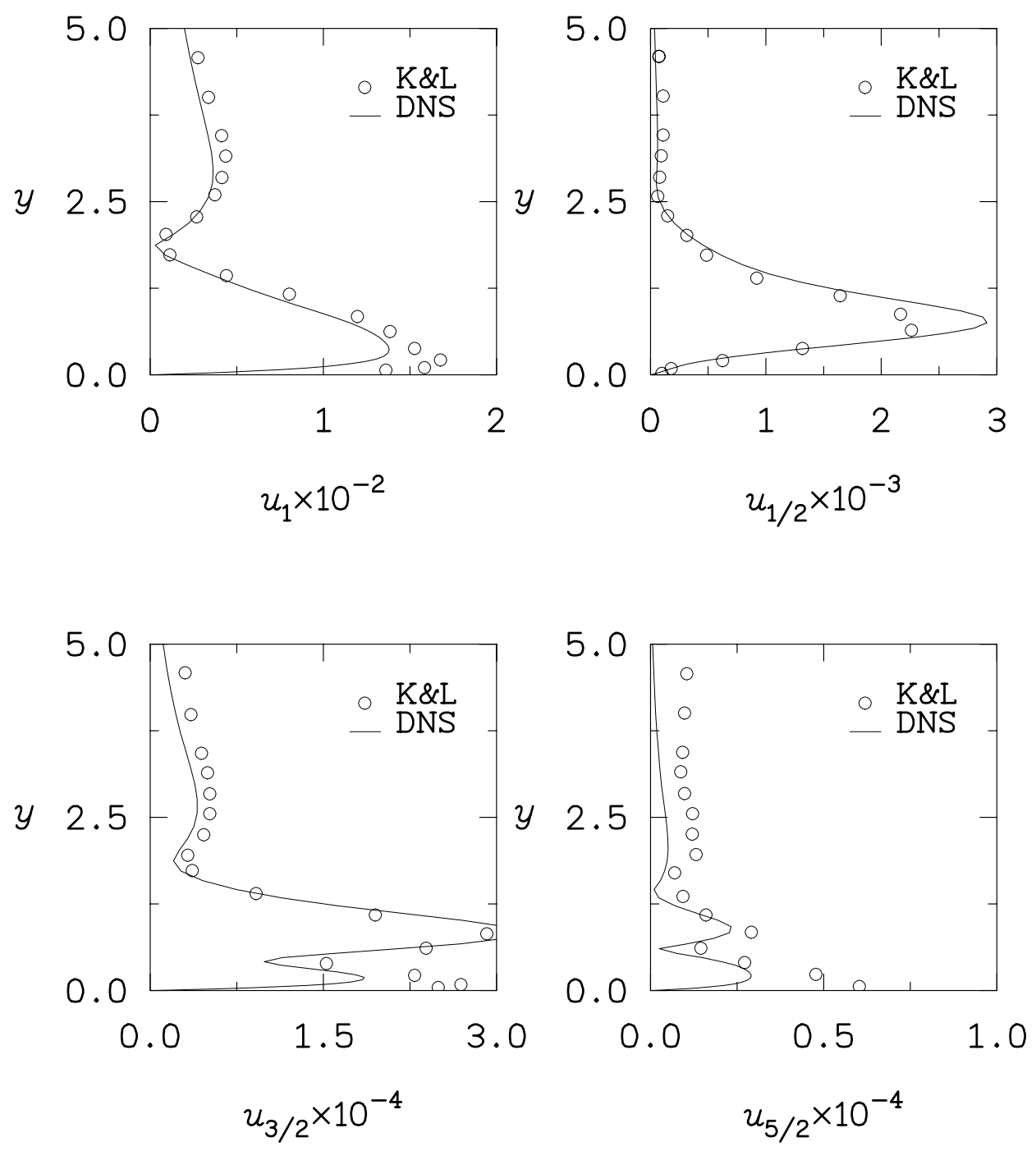

Figure 8. Streamwise disturbance profiles at the downstream location where locally $R_{\delta^{*}}=$ 1049 for subharmonic breakdown. $\left[u_{n}=u_{n F}\right]$ 


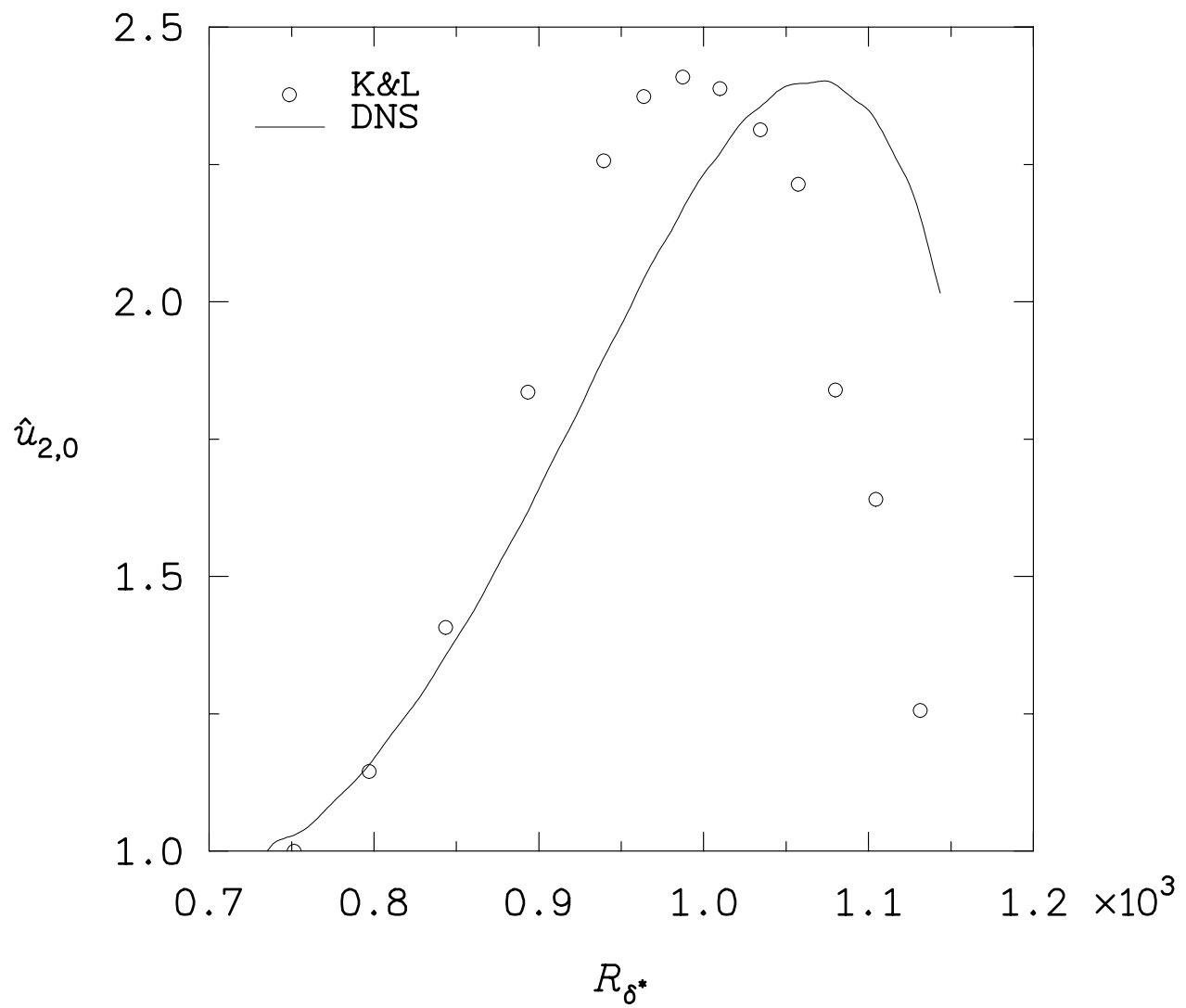

Figure 9. Amplitude growth with downstream distance for only primary waves at $y=0.26 \delta$. 


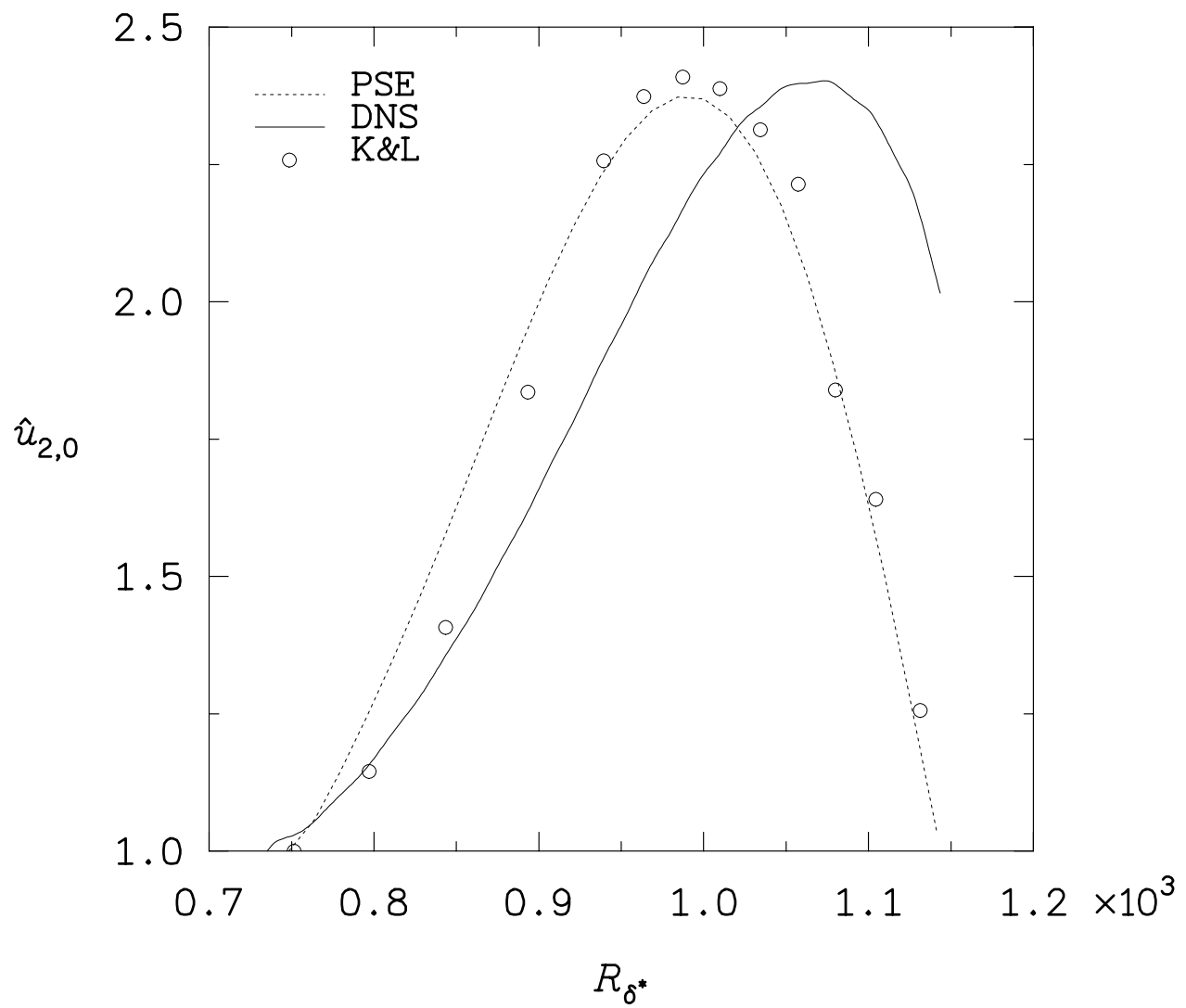

Figure 10. Amplitude growth with downstream distance for the previous primary waves and a primary wave computed with a frequency and pressure-gradient varitation at $y=0.26 \delta$. 


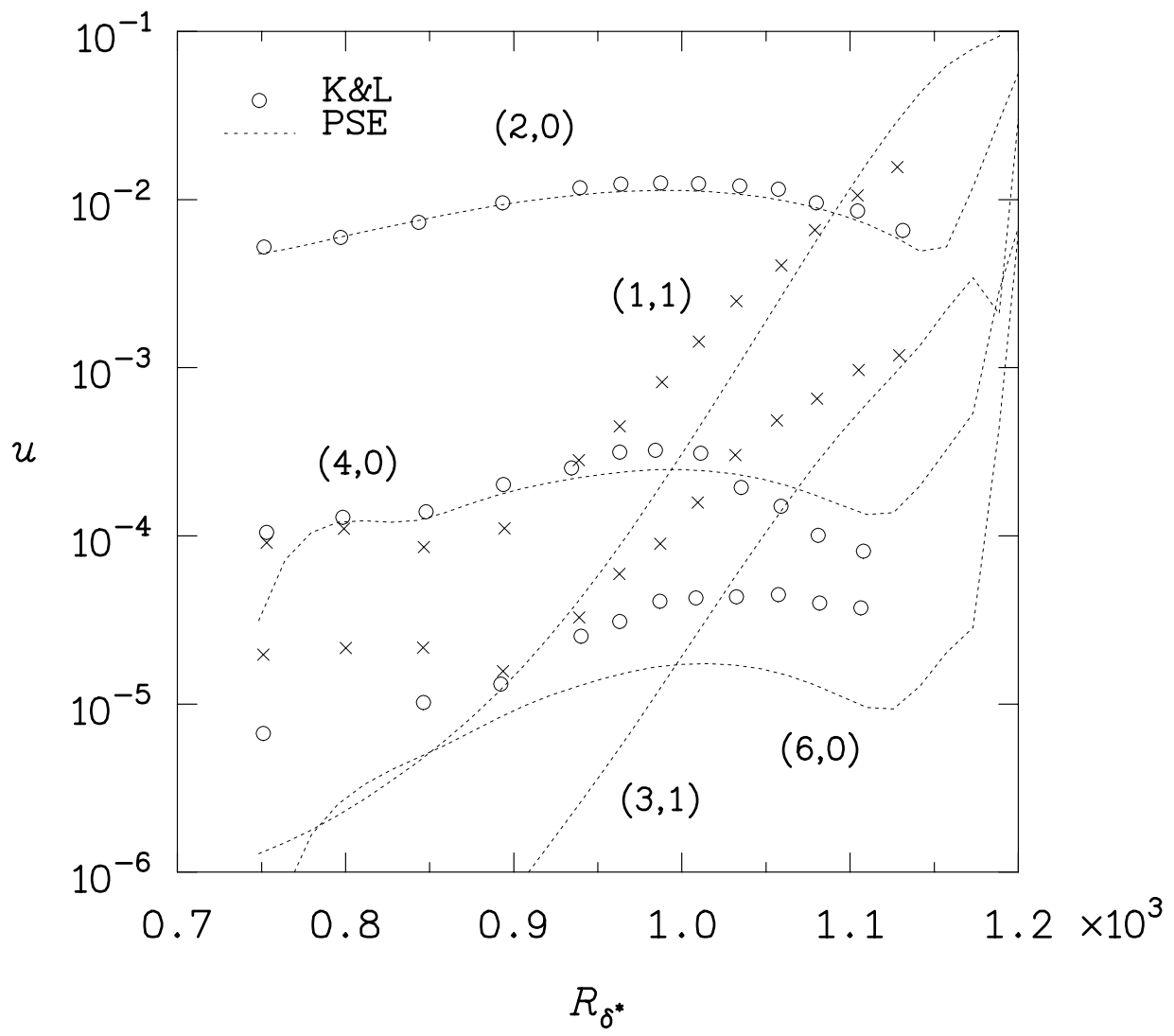

Figure 11. Amplitude growth with downstream distance for the primary wave $[F=(2,0)]$, subharmonic $[F / 2=(1,1)]$, and various higher harmonics $[2 F=(4,0), 3 F=(6,0), 3 F / 2=$ $(3,1)]$ at $y=0.26 \delta$. 


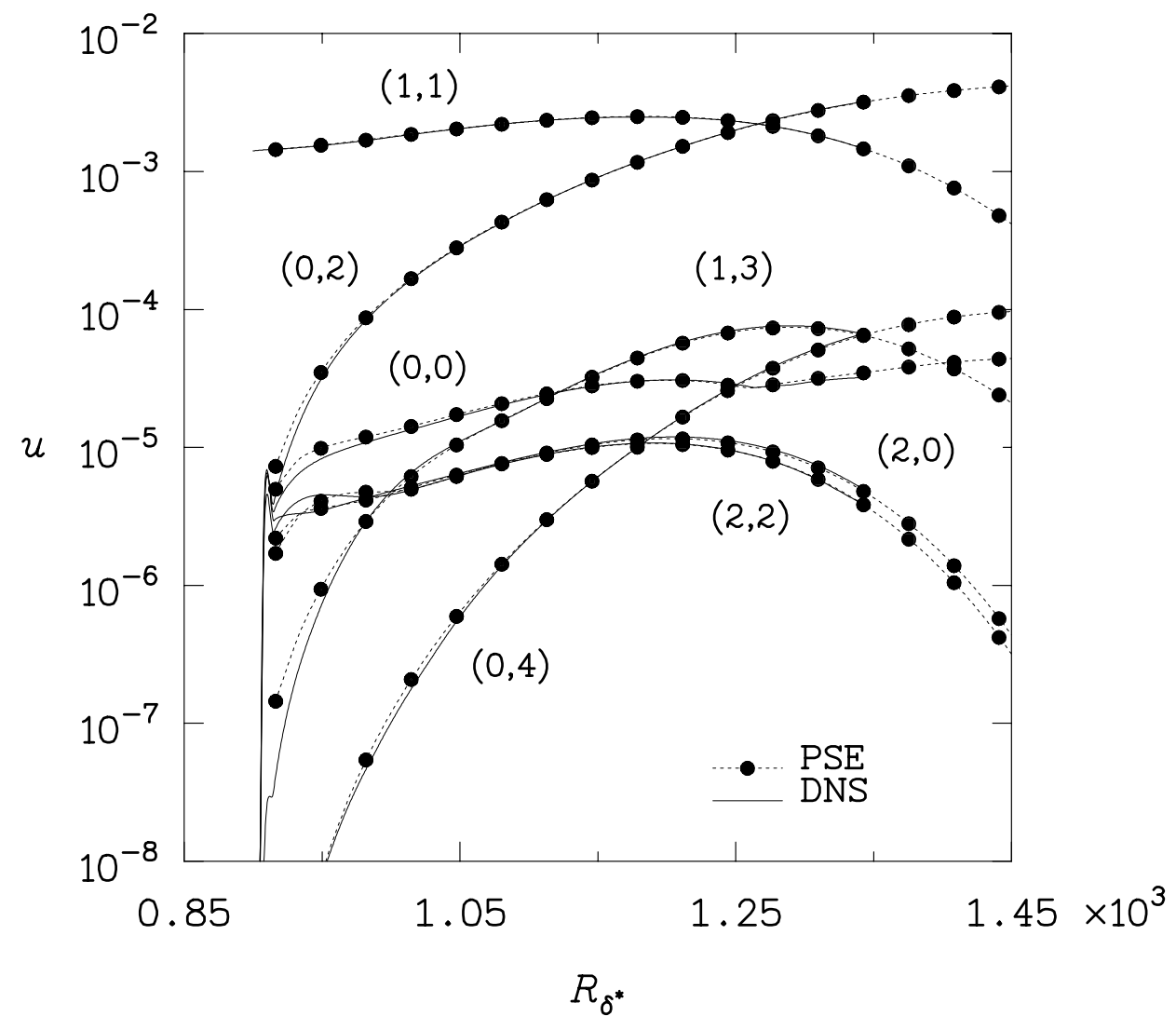

Figure 12. Amplitude growth with downstream distance, from an oblique-wave pair, each with amplitude $A_{1,1}^{o}=0.001$. 

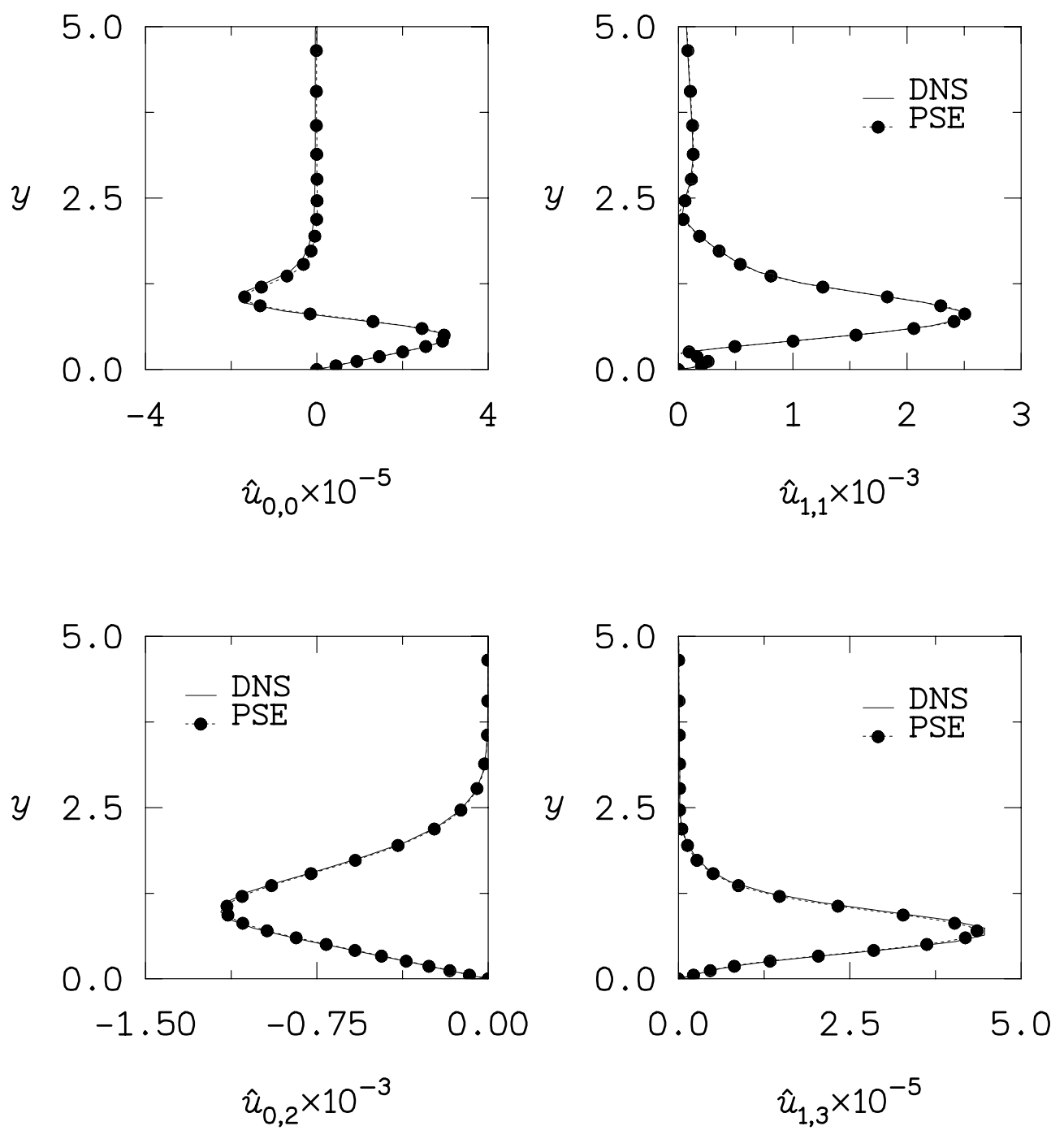

Figure 13. Streamwise disturbance velocity profiles at a downstream location where locally $R_{\delta^{*}}=1178$ for the oblique-wave case with $A_{1,1}^{o}=0.001$. 


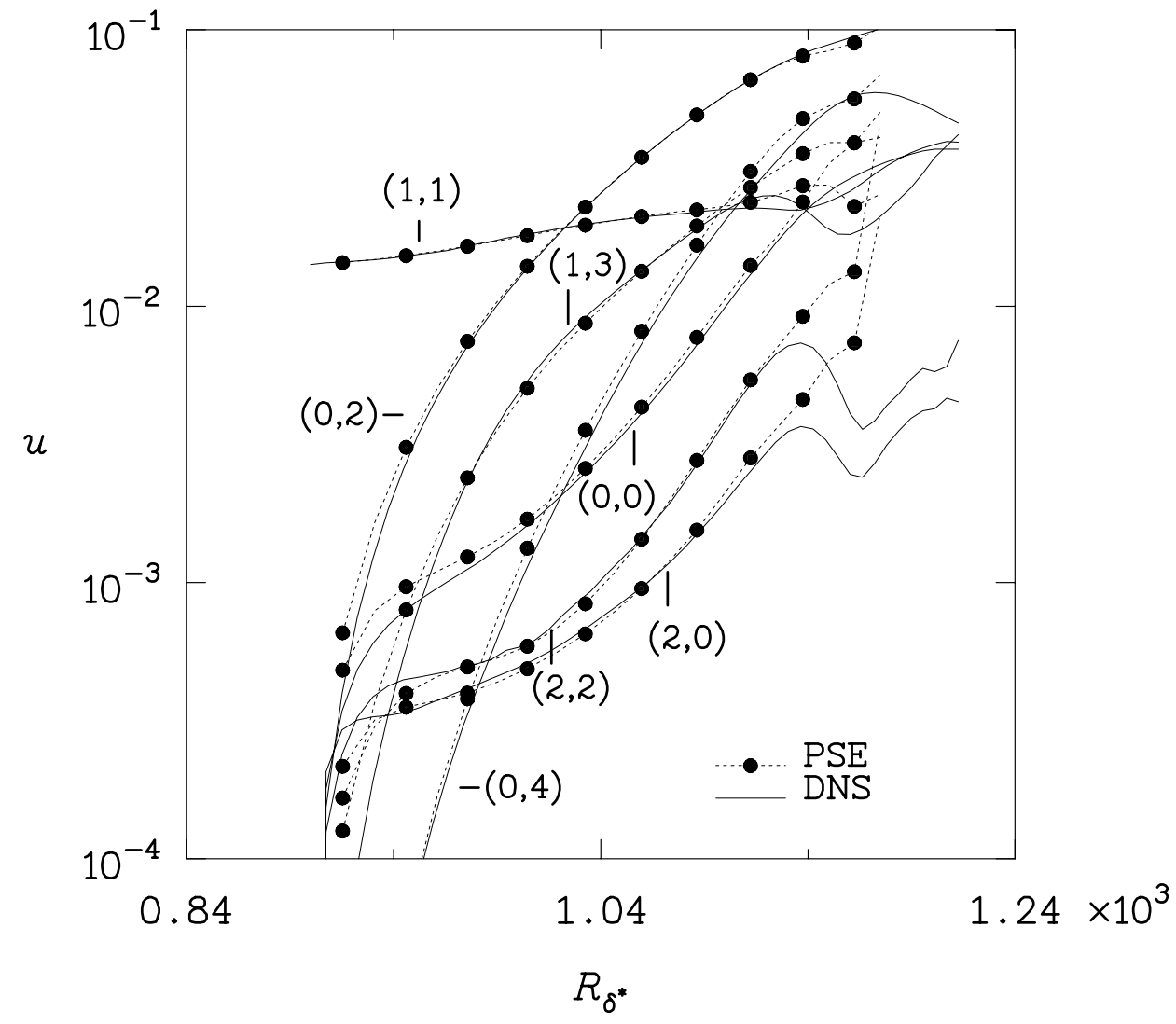

Figure 14. Amplitude growth with downstream distance, from an oblique-wave pair, each with amplitude $A_{1,1}^{o}=0.01$. 